\title{
Article \\ Combining HDAC and MEK Inhibitors with Radiation against Glioblastoma-Derived Spheres
}

\author{
Eno I. Essien ${ }^{1,2}\left(\mathbb{D}\right.$, Thomas P. Hofer ${ }^{3}\left(\mathbb{D}\right.$, Michael J. Atkinson ${ }^{4}$ and Nataša Anastasov ${ }^{1,2, *} \mathbb{C}$ \\ 1 Institute of Biological and Medical Imaging, Helmholtz Center Munich, German Research Center for \\ Environmental Health, 85764 Neuherberg, Germany; eno.essien@helmholtz-muenchen.de \\ 2 Institute of Radiation Biology, Helmholtz Center Munich, German Research Center for Environmental Health, \\ 85764 Neuherberg, Germany \\ 3 Immunoanalytics Research Group Tissue Control of Immunocytes \& Core Facility, German Research Center \\ for Environmental Health, 81377 Munich, Germany; hofer@helmholtz-muenchen.de \\ 4 Chair of Radiation Biology, Technical University of Munich, 80333 Munich, Germany; m.j.atkinson@tum.de \\ * Correspondence: natasa.anastasov@helmholtz-muenchen.de; Tel.: +49-893-187-3798; Fax: +49-893-187-3378
}

Citation: Essien, E.I.; Hofer, T.P.; Atkinson, M.J.; Anastasov, N Combining HDAC and MEK Inhibitors with Radiation against Glioblastoma-Derived Spheres. Cells 2022, 11, 775. https://doi.org/ 10.3390/cells 11050775

Academic Editors: Javier S.

Castresana and Bárbara Meléndez

Received: 31 January 2022

Accepted: 18 February 2022

Published: 23 February 2022

Publisher's Note: MDPI stays neutral with regard to jurisdictional claims in published maps and institutional affiliations.

Copyright: (C) 2022 by the authors. Licensee MDPI, Basel, Switzerland. This article is an open access article distributed under the terms and conditions of the Creative Commons Attribution (CC BY) license (https:// creativecommons.org/licenses/by/ $4.0 /)$.

\begin{abstract}
Glioblastoma stem-like cells (GSLCs) in glioblastoma limit effective treatment and promote therapeutic resistance and tumor recurrence. Using a combined radiation and drugscreening platform, we tested the combination of a histone deacetylase inhibitor (HDACi) and MAPK/ERK kinase inhibitor (MEKi) with radiation to predict the efficacy against GSLCs. To mimic a stem-like phenotype, glioblastoma-derived spheres were used and treated with a combination of HDACi (MS-275) and MEKi (TAK-733 or trametinib) with 4 Gy irradiation. The sphere-forming ability after the combined radiochemotherapy was investigated using a sphere formation assay, while the expression levels of the GSLC markers (CD44, Nestin and SOX2) after treatment were analyzed using Western blotting and flow cytometry. The combined radiochemotherapy treatment inhibited the sphere formation in both glioblastoma-derived spheres, decreased the expression of the GSLC markers in a cell-line dependent manner and increased the dead cell population. Finally, we showed that the combined treatment with radiation was more effective at reducing the GSLC markers compared to the standard treatment of temozolomide and radiation. These results suggest that combining HDAC and MEK inhibition with radiation may offer a new strategy to improve the treatment of glioblastoma.
\end{abstract}

Keywords: glioblastoma; glioblastoma-derived spheres; HDAC inhibitor; MEK inhibitor; radiation; combination therapy

\section{Introduction}

Glioblastoma (GB), a grade IV astrocytoma, is one of the most aggressive primary brain tumors. Despite the adoption of a standard therapy combining surgical resection, radiotherapy and chemotherapy with temozolomide (TMZ), the poor prognoses of patients with GB have failed to improve, with a median survival of only 14.6 months [1]. One factor that limits the success of GB therapy is the presence of a sub-population of glioblastoma stem-like cells (GSLCs) within the tumor [2]. These GSLCs possess characteristics of tissue stem cells, including the ability to self-renew and to generate further malignant progeny [3]. GSLCs are considered to be drug and radiation resistant, as well as promote tumor angiogenesis and tumor recurrence, all of which hinder the effective treatment of GB $[2,4,5]$. A therapeutic strategy that improves the control of GSLCs offers an opportunity to improve treatment outcomes for GB.

One approach against GB may be to inhibit the MAPK/ERK kinase (MEK), situated downstream of the RAS-RAF-MEK-MAPK pathway, stimulating the proliferation and 
survival of GSLCs [6,7]. The MAPK pathway is activated by a series of phosphorylation events that can be targeted through MEK, the downstream activator of MAPK. Trametinib and TAK-733 are small-molecule-selective MEK inhibitors with antitumor activity in cancers, such as gliomas, multiple myeloma, melanoma and triple-negative breast cancer [8-12]. Trametinib has recently been applied in clinical studies of brain tumors, suggesting that it has the ability to cross the blood-brain barrier (BBB) $[13,14]$. Although these MEK inhibitors (MEKi) were found to have good safety profiles in clinical trials, they only exhibited limited antitumor activity as single agents due to resistance $[10,11,15]$. Therefore, MEKi in combination with other anti-cancer agents may lead to more effective therapies. For instance, we recently showed that the effect of TAK-733 on reducing the migratory potential of breast cancer cells was enhanced by 4 Gy irradiation [12].

Various studies reported enhanced antitumor activity when combining MEK and histone deacetylases inhibitors (HDACis) [16-19]. HDACs are enzymes that decrease acetylation and are epigenetic regulators of gene expression that contribute to the pathogenesis of cancers, such as GB. Therefore, HDACis are considered promising therapeutics for cancer treatment $[20,21]$. For example, it was shown that the HDACis Entinostat (MS-275) and trichostatin A (TSA) inhibited the formation of GB-derived neurospheres and reduced GB xenograft growth [22]. It is also noteworthy that MS-275 demonstrated the ability to cross the $\mathrm{BBB}$ in vivo via increased acetylation of histone $\mathrm{H} 3$ in brain tissue of syngeneic rats after intratumoral injection [23]. Several other HDACis showed promising results in preclinical studies but few made it to clinical trials due to limited efficacy for GB therapy as a single treatment [24]. However, the combined effect of HDACis with other anticancer agents seems more promising and is being investigated in preclinical and clinical combination studies [24-26].

The combination of an HDACi and a MEKi showed promising results in other cancers $[16,27,28]$ but, to date, has not been explored regarding GB. Clinical studies of GB revealed that monotherapy with newly discovered therapeutics failed to improve survival [29]. In addition, tumor heterogeneity, as well as multiple dysregulated pathways, characterizes GB; therefore, a combination treatment strategy was proposed as the most effective approach to improve therapy [30]. Thus, the aim of our study was to investigate the potential effects of combining the HDACi MS275 and the MEKi TAK-733 or trametinib with radiation using human GB-derived spheres that mimic a stem-like phenotype. A panel of markers (ALDH1A1, CD133, CD44, Nestin and SOX2) that is associated with stemness to drive tumorigenesis was used to measure and predict the effect of this radiochemotherapy approach against GSLCs. The results demonstrated that this multimodal therapeutic strategy is promising and could offer an opportunity to improve the treatment and survival of GB patients.

\section{Materials and Methods}

\subsection{Growth and Maintenance of Cell Lines}

The human GB cell lines U87 and U251 were obtained from Sirion Biotech GmbH (Martinsried, Germany). Both GB cell lines were cultured in high-glucose Dulbecco's Modified Eagle Medium (DMEM) with GlutaMAX ${ }^{\mathrm{TM}}-\mathrm{I}, 4.5 \mathrm{~g} / \mathrm{L}$ glucose, pyruvate and $10 \%$ FCS. The cell lines were maintained under standard incubator conditions at $37^{\circ} \mathrm{C}$ in a humidified atmosphere containing $5 \% \mathrm{CO}_{2}$. In addition, the U87 and U251 cell lines were cultured as spheres (U87-sph and U251-sph) in a serum-free DMEM/F12 high-glucose medium with GlutaMAX ${ }^{\mathrm{TM}}-\mathrm{I}, 4.5 \mathrm{~g} / \mathrm{L}$ glucose and pyruvate to induce a stem-like phenotype. The stem cell supplements were $1 \times$ B27 supplement (Gibco Life Technologies, Darmstadt, Germany), 1× N2 supplement (Gibco Life Technologies, Darmstadt, Germany), $1 \times$ Penicillin-Streptomycin (Sigma-Aldrich, Steinheim, Germany), $1 \times \mathrm{D}-(+)$-Glucose Solution $45 \%$ in $\mathrm{H}_{2} \mathrm{O}$ (Sigma-Aldrich, Steinheim, Germany), $20 \mathrm{ng} / \mathrm{mL}$ Epidermal Growth Factor (EGF) Human+ (Sigma-Aldrich, Steinheim, Germany), and 
20 ng/mL Fibroblast Growth Factor (FGF) Basic Human+ (Sigma-Aldrich, Steinheim, Germany). The glioblastoma-derived spheres were cultured for at least eight passages and the expressions of stem cell markers were analyzed before they were used for experiments. The cell lines were checked for mycoplasma contamination using the MycoAlert Detection Kit (Lonza Group Ltd., Basel, Switzerland), while the cell line authentication was done via genetic profiling using the PowerPlex ${ }^{\circledR} 21$ System (Eurofins, Ebersberg, Germany).

\subsection{Web Database Analysis of $G B$}

The GEPIA web server (http:/ /gepia.cancer-pku.cn, accessed on 19 January 2021) [31] was used to obtain the GB stem cell marker expression. Box plots were downloaded to compare the CD44, Nestin and SOX2 expression levels between non-cancerous (207 samples) and GB tumor samples (163 samples) from the TCGA and GTEx databases.

\subsection{Immunofluorescence Assay}

The assay was performed by seeding $4 \times 10^{5}$ cells (U87, U87-sph, U251 and U251sph) on microscopic slides placed in 4 -well chambered plates and left overnight. The cells were fixed the next day in $4 \%$ paraformaldehyde (PFA) for $15 \mathrm{~min}$ at room temperature, followed by 3 washes with PBS. Permeabilization was done with $0.2 \%$ Triton X-100 for 5 min (only for the intracellular staining of ALDH1A1, SOX2 and Nestin). The cells were washed 2 times in PBS and blocked in 1\% BSA and $0.15 \%$ glycine in PBS for $1 \mathrm{~h}$ at room temperature. After blocking, incubation was done overnight at $4{ }^{\circ} \mathrm{C}$ with the following antibodies; ALDH1A1 (36671, 1:100; Cell Signalling Technology, Danvers, MA, USA), CD133 (Ab16518, 1:100; Abcam, Cambridge, UK), CD44 (3570s, 1:1000; Cell Signaling Technology, Danvers, MA, USA), SOX2 (3579s, 1:500; Cell Signaling Technology, Danvers, MA, USA) and Nestin (MA1-110, 1:500; Thermo Fisher Scientific, Darmstadt, Germany). The next day, the cells were washed with PBS and incubated with the secondary antibody mix for $1 \mathrm{~h}$ at room temperature. The secondary antibodies were Cy3-Goat Anti-Rabbit (A10520, Red, 1:300) and Alexa Flour 488-Goat Anti-Mouse (A11029, Green, 1:200, Life Technologies, Eugene, OR, USA). The cells were washed 3 times with PBS and cell nuclei were stained with DAPI. Imaging of cells was performed at a magnification of $40 \times$ using a Keyence BZ 9000 fluorescence microscope (Keyence, Frankfurt, Germany).

\subsection{Treatment with HDACi, MEKi and Radiation}

The GB-derived spheres were treated according to previously published protocols [26,32]. Briefly, the following conditions were applied: (1) $1 \mu \mathrm{M}$ of the HDACi MS-275 (S1053; purchased from Selleck Chemicals), (2) $1 \mu \mathrm{M}$ of the MEKi TAK-733 (S2617; purchased from Selleck Chemicals), (3) $1 \mu \mathrm{M}$ trametinib (S2673; purchased from Selleck Chemicals), (4) a combination of $1 \mu \mathrm{M}$ MS-275 plus $1 \mu \mathrm{M}$ TAK-733 or (5) a combination of $1 \mu \mathrm{M}$ MS-275 plus $1 \mu \mathrm{M}$ trametinib. Where specified, the GBderived spheres were treated with the standard compound TMZ at $50 \mu \mathrm{M}$ (SC-203292; purchased from Santa Cruz Biotechnology, Dallas, TX, USA) for comparison. All compounds were diluted to give a final concentration of $1 \% v / v$ DMSO and the controls were treated with $1 \% v / v$ DMSO. The GB-derived spheres were treated $72 \mathrm{~h}$ after seeding the cells to allow time for sphere formation. After $24 \mathrm{~h}$ of compound treatment, the spheres were irradiated at room temperature with X-rays using an X-Strahl RS225 radiation device (X-Strahl LTD, Camberlay, UK). The 4 Gy irradiation dose was delivered at a rate of $0.824 \mathrm{~Gy} / \mathrm{min}$ using a $3 \mathrm{~mm}$ aluminum filter. The sham irradiated controls were handled under the same conditions but were not exposed to radiation. 


\subsection{Cell Viability Assay}

Cell viability was tested using CellTiter-Glo ${ }^{\circledR}$ Luminescent Cell Viability Assays (Cat.Nr. G75751) according to the manufacturer's instructions (Promega, Madison, WI, USA) and previously published data [12]. U87-sph and U251-sph cells were seeded at $3 \times 10^{5}$ cells per well in 12-well ultra-low-attachment (ULA) plates (Corning, NY, USA). Since the spheres were dissociated into single cells for seeding, the spheroid formation was allowed for $72 \mathrm{~h}$. This was followed by treatment with increasing concentrations of MS-275, TAK-733, trametinib and TMZ at $1 \mu \mathrm{M}, 10 \mu \mathrm{M}$ and $50 \mu \mathrm{M}$. Irradiation was done $24 \mathrm{~h}$ after treatment at $4 \mathrm{~Gy}$ and incubated for an additional $72 \mathrm{~h}$ to have a final time point for analysis of $96 \mathrm{~h}$. After this period, the spheres were dissociated with Accutase and counted to re-seed them at $1 \times 10^{4}$ in 96-well ULA plates. The cell CellTiter-Glo ${ }^{\circledR}$ reagent was added after $72 \mathrm{~h}$ under cell culture conditions. Incubation was done for $10 \mathrm{~min}$ at room temperature before recording the luminescence at $560 \mathrm{~nm}$ emission using an infinite M200 plate reader (TECAN, Maennedorf, Switzerland). The measurements were performed in quadruplicates for three independent experiments.

\subsection{Sphere Formation Assay}

After $72 \mathrm{~h}$ of treatment (described in Section 2.4) with both compounds and radiation, the GB-derived spheres were harvested and reseeded in triplicates at 200 cells per well in 96-well ULA plates (Corning Inc., Corning, NY, USA) and cultured for at least 2 weeks. The images of spheres in each well were taken using an Operetta imaging system (PerkinElmer, Waltham, MA, USA). The images were taken using the brightfield channel and $10 \times$ magnification, while the sphere number per well was counted manually. The sphere formation rate was determined by the number of spheres formed divided by the total number of starting cells.

\subsection{Western Blot Analysis}

Cell pellets were collected after $72 \mathrm{~h}$ of compound and radiation treatment (described in Section 2.4). Lysing, protein extraction and immunoblotting were performed as previously described [12]. The target proteins of the GSLC markers were detected with the same antibodies used for immunofluorescence staining listed in Section 2.3, including Acetyl-Histone H3 (9677) and Histone H3 (4499, 1:1000; Cell Signaling, Danvers, MA, USA), MAPK (9101) and phospho-MAPK (9102, Cell Signaling, Danvers, MA, USA), with $\beta$-Actin (A5441, 1:20,000; Sigma Aldrich, Steinheim, Germany) as the loading control. The secondary antibodies were horseradish peroxidase-conjugated anti-mouse (A16066, 1:20,000) and anti-rabbit (A16096, 1:10,000; Invitrogen, Carlsbad, CA, USA). The bands of the secondary bound antibodies were detected using enhanced chemiluminescence (ECL) (Amersham, England) reagents. The luminescent signal was detected and captured using an Alpha Innotech ChemiImager system (Biozym, Hessisch Oldendorf, Germany). The GSLC markers ALDH1A1 and CD133 were not detected well using Western blotting and, therefore, excluded from further analysis.

In the case of reprobing, the membranes were stripped with Restore PLUS Western Blot Stripping Buffer (Thermo Scientific, Rockford, IL, USA) for $15 \mathrm{~min}$ at room temperature. For the quantification of the band intensities, the Image-J image analysis software [33] was used.

\subsection{Flow Cytometry Analysis}

The cells were harvested $72 \mathrm{~h}$ after treatment (described in Section 2.4) and washed once with PBS. Afterward, the cells were blocked in Anti-Hu Fc Receptor Binding Inhibitor (14916173, 1:10 in PBS, Invitrogen, Carlsbad, CA, USA) for $10 \mathrm{~min}$ at $4{ }^{\circ} \mathrm{C}$ and then washed with PBS. Live-dead staining of cells was done via incubation with a Zombie Aqua ${ }^{\mathrm{TM}}$ Fixable Viability Kit (423101, 1:100 in PBS, BioLegend, San Diego, CA, USA) for $30 \mathrm{~min}$ at room temperature. After washing in FACS buffer $(0.5 \%$ BSA in PBS), the cells were stained with BV-785-conjugated CD44 (103041, 1:100; Biole- 
gend, San Diego, CA, USA) diluted in FACS buffer and incubated for $30 \mathrm{~min}$ at $4{ }^{\circ} \mathrm{C}$. Next, the cells were fixed in $1 \times$ fixation buffer for $30 \mathrm{~min}$ at room temperature and washed with $1 \times$ permeabilization buffer using the Foxp3/Transcription Factor Staining Buffer Set (5523, Invitrogen, Carlsbad, CA, USA). This was followed by incubation with an antibody mix of APC-Conjugated Nestin (MA5-23650, 1:100; Thermo Fisher Scientific, Darmstadt, Germany) and PerCP-Cy5.5-Conjugated SOX2 (561506, 1:50; BD Biosciences, San Diego CA, USA) in $1 \times$ permeabilization buffer for $1 \mathrm{~h}$ at room temperature. Additionally, staining with PerCP-Cy ${ }^{\mathrm{TM}} 5.5$ Mouse IgG1 k Isotype Control (550795, 1:50; BD Biosciences, San Diego CA, USA) was applied. The cells were washed twice in $1 \times$ permeabilization buffer, resuspended in PBS and passed through a $40 \mu \mathrm{m}$ mesh filter into FACS tubes to remove clumped cells and obtain a singlecell suspension.

The cells were analyzed via flow cytometry using a CytoFLEX LX Flow Cytometer and CytExpert software (Beckman Coulter, Krefeld, Germany). The CytoFLEX LX instrument has a capacity for 21 fluorescence detections and is equipped with a $355 \mathrm{~nm}$ (UV) laser, $405 \mathrm{~nm}$ (violet) laser, $488 \mathrm{~nm}$ (blue) laser, $561 \mathrm{~nm}$ (yellow-green) laser, $638 \mathrm{~nm}$ (red) laser and $808 \mathrm{~nm}$ (infrared) laser. Fluorescence and side scatter light of the CytoFLEX LX were delivered via fiber optics to avalanche photodiode detector arrays, while the emission profiles were collected using reflective optics and single-transmission band-pass filters. Unstained cells were used to set the voltages, while compensation beads (BD Biosciences, San Diego, CA, USA) were used for compensation to correct for spectral overlap across the fluorescent channels. The gating strategy to set a cut-off for negative and positive populations was done using two gating controls. First, unstained cells were used to set negative and positive gates, while the fluorescence minus one (FMO) control was used to address any spillover-induced background [34]. Additionally, an isotype control for SOX2 was included to set gates against non-specific antibody binding. The gating region on the controls was set to contain less than $1 \%$ of the cells for both single- and double-positive populations (Figures S4, S5 and S7).

\subsection{Statistical Analysis}

All experiments consisted of three biological replicates unless otherwise indicated and the data represent the mean \pm standard error of the mean (SEM). The differences in mean values between two groups (control and treated) were compared using Student's t-tests and statistical significance defined with $p$-values as follows: ${ }^{*} p \leq 0.05,{ }^{* *} p \leq 0.01$ and *** $p \leq 0.001$.

\section{Results}

\subsection{Influence of Radiation Alone on Specific GSLC Marker Expression}

The expression of CD44, Nestin and SOX2 in the GB samples and adjacent noncancerous brain tissue were examined using the GEPIA webserver to interrogate publicly available gene expression databases from the TCGA and GTEx projects. Within the matched TCGA normal and GTEx data, 163 GB tumor samples and 207 non-cancerous samples were analyzed. The gene expression of CD44, Nestin and SOX2 were all significantly higher in GB tumor samples than in the non-cancerous tissue samples (Figure 1a).

The protein expressions of CD44, Nestin and SOX2 were further detected in the U87 and U251 human GB cell lines. All three markers could be detected in both cell lines, except for SOX2 not detected in U87. Additionally, the effect of radiation alone was investigated and it was observed that there was no beneficial effect on the expression of the GSLC markers $72 \mathrm{~h}$ after $4 \mathrm{~Gy}$ radiation in vitro (Figure 2b). Since Nestin, CD44 and SOX2 are associated with stemness in GB, their elevated levels in the GB tumor samples suggested that these markers may drive the progression and radioresistance of GB. 
(a) Glioblastoma stem cell marker expression in GB (TCGA and GTEx database)
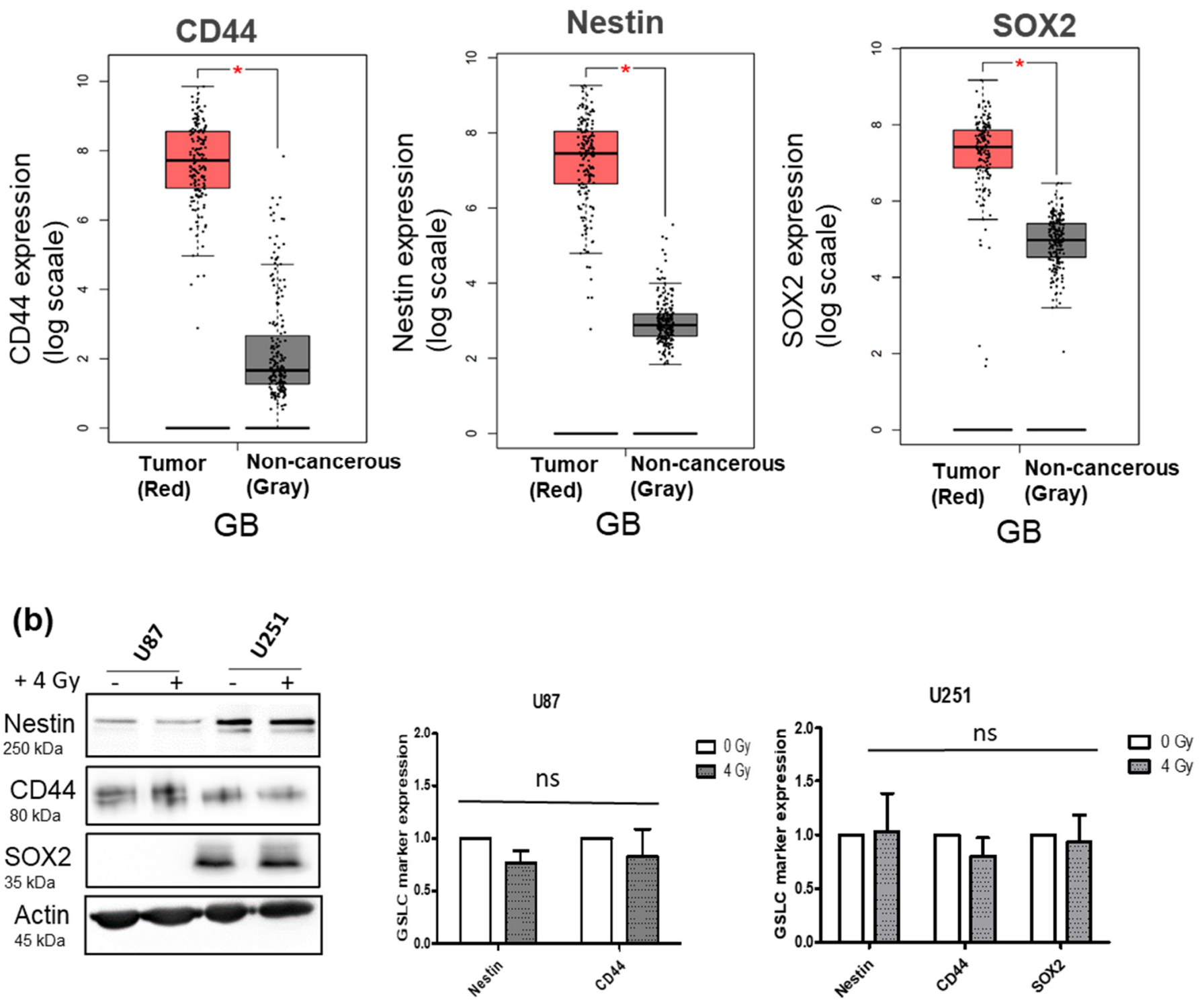

Figure 1. Glioblastoma stem cell marker expression in GB (from TCGA and GTEx database) and GB cell lines: (a) TCGA database comparison of GB stem cell marker (CD44, Nestin and SOX2) expression between GB (red; 163 samples) and non-cancerous tissue (gray; 207 samples). Box plots derived from matching TCGA normal and GTEx data downloaded via the GEPIA webserver ( ${ }^{*} p$-value $\left.<0.05\right)$. (b) Protein expression of CD44, Nestin and SOX2 in U87 and U251 GB cell lines and the effect of 4 Gy irradiation. Data represent mean values of three replicates and the error bars \pm SEM $(n=3$; ns-nonsignificant).

\subsection{Induced GSLC Marker Expression by GB-Derived Spheroid Culture in Serum-Free Medium}

To determine whether a stem-like phenotype was induced by the spheroid culture in the serum-free medium in vitro, co-immunofluorescence staining of GSLC markers was performed. After eight passages in the serum-free medium, the GB-derived spheres and their parental cell lines were immunostained to compare the GSLC marker levels in both the serum-free and serum-containing culture conditions. The results showed an increased co-expression of CD133 and CD44, ALDH1A1 and Nestin or SOX2 and Nestin in U87-sph cells (grown in serum-free medium) compared to U87 cells (grown in medium containing 
10\% FCS) (Figure 2b). Most of the U251-sph cells expressed CD133, CD44, Nestin and SOX2, while fewer U251 cells expressed these stem cell markers. However, ALDH1A1 was not detected in both. Similar results were observed in the U87 parental cells and U87-sph cells. More cells expressed all GSLC markers in U87-sph compared to U87 cells (Figure 2d). These results implied that the culture of the GB cell lines in the serum-free medium could enrich GSLC marker expression.

(a)
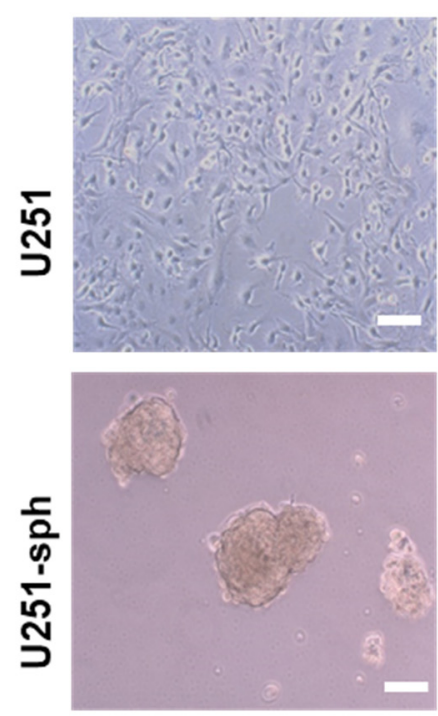

(c)
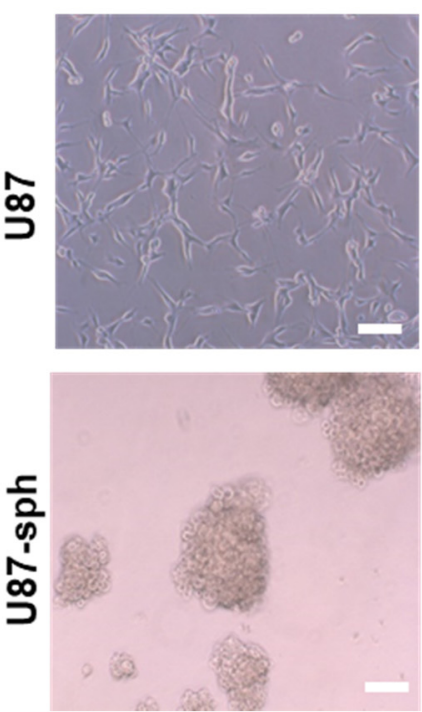

(b)
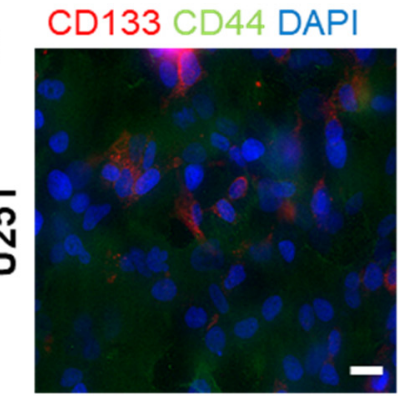

ALDH1A1 Nestin DAPI SOX2 Nestin DAPI
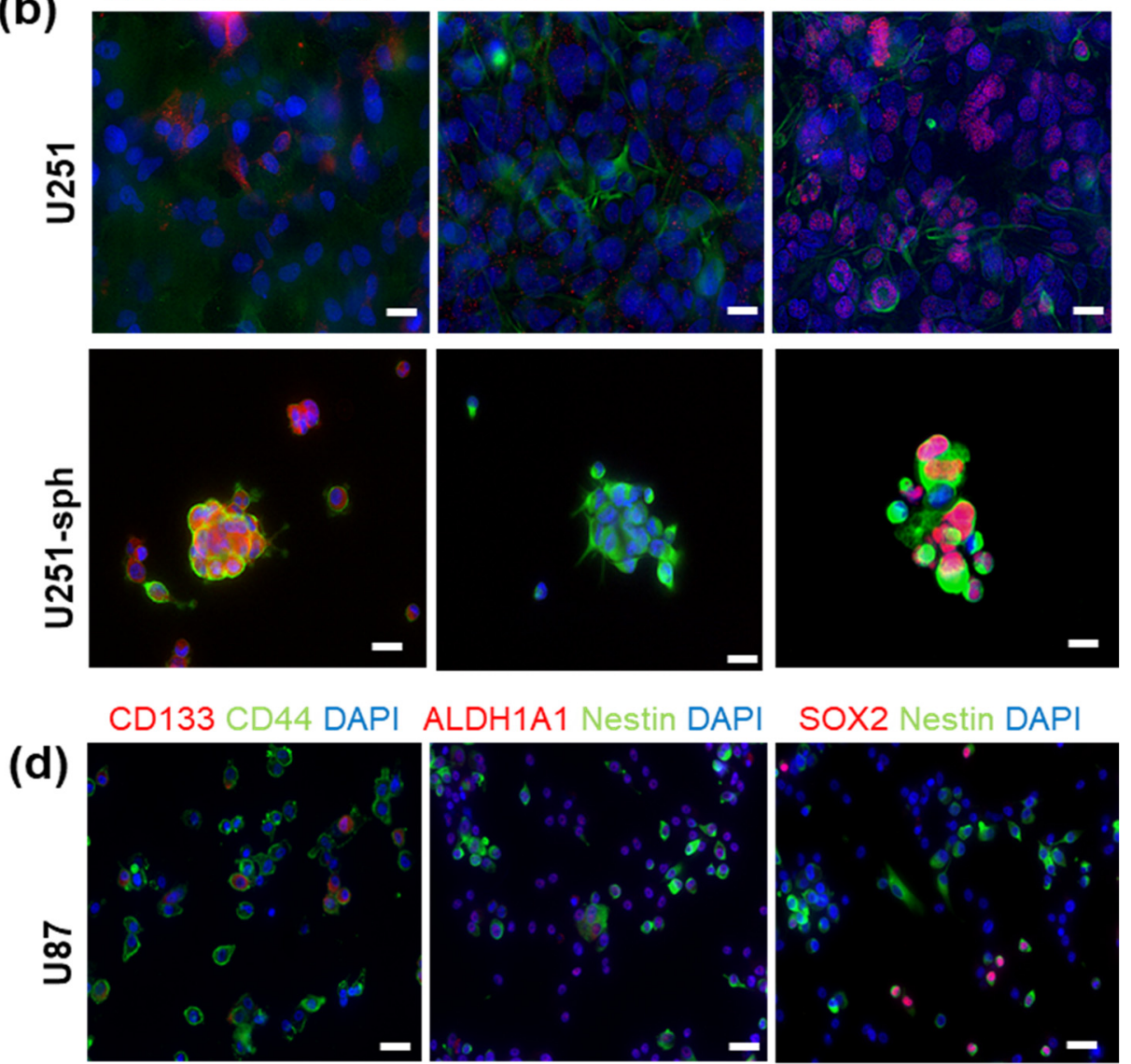

SOX2 Nestin DAPI
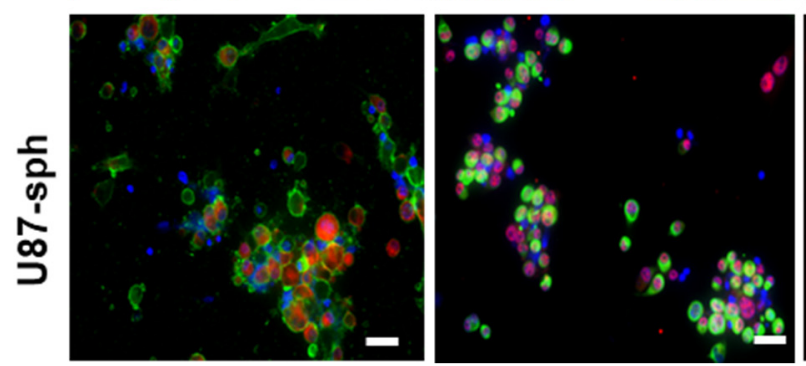

Figure 2. Induction of the stem-like phenotype in U251 and U87 cell lines by a spheroid culture. (a) Representative images of the morphology of U251 (cultured in the medium containing 10\% FCS) and U251-sph (cultured in the serum-free medium; scale bar: $100 \mu \mathrm{m}$ ). (b) Representative images of co-immunofluorescence staining of U251 and U251-sph. The U251-sph cells showed an increased dual expression of the GSLC markers CD133 (red), CD44 (green), Nestin (green) and SOX2 (red) compared to the U251 cells. Nuclei were stained with DAPI (blue). Scale bar: $100 \mu \mathrm{m}$. (c) Representative images of the morphology of U87 (cultured in the medium containing 10\% FCS) and U87-sph (cultured in the serum-free medium; scale bar: $100 \mu \mathrm{m}$ ). (d) Representative images of immunofluorescence staining of U87 and U87-sph. The U87-sph cells showed an increased co-expression of the GSLC markers CD133 (red), CD44 (green), ALDH1A1 (red), Nestin (green) and SOX2 (red) compared to the U87 cells. Nuclei were stained with DAPI (blue). Scale bar: $100 \mu \mathrm{m}$. 


\subsection{HDAC and MEK Inhibitors with 4 Gy Radiation Decreased Cell Viability and Sphere Formation}

To identify the most potent concentration of the inhibitors in combination with 4 Gy radiation, U87-sph and U251-sph were treated in an increasing concentration range of 1,10 and $50 \mu \mathrm{M}$ and the cell viability was determined $72 \mathrm{~h}$ after radiation exposure. For both U251-sph and U87-sph, the viability was significantly decreased by the HDACi (MS-275) or the MEKi (TAK-733 or trametinib) alone and with 4 Gy irradiation in a concentration-dependent manner (Figure S2a-f). Interestingly, the viability of the U251-sph cells was significantly increased by treatment with the standard compound TMZ, even at $50 \mu \mathrm{M}$; when combined with radiation, no change was observed in comparison to the 4 Gy irradiation alone (Figure S2d-f). This suggested that the HDAC and MEK inhibitors were more potent at lower concentrations $(1 \mu \mathrm{M})$ compared to the standard compound TMZ $(50 \mu \mathrm{M})$ used for radiochemotherapy of GB.

The activities of the inhibitors were additionally validated in both GB-derived spheres. Treatment with MS-275 at 1 and $10 \mu \mathrm{M}$ increased the amount of acetylated histone $\mathrm{H} 3$, indicating that HDACs were inhibited (Figure S1a). The inhibitory effects of TAK-733 and trametinib at 1 and $10 \mu \mathrm{M}$ were confirmed by low amounts of activated MAPK (pMAPK) compared to MAPK (Figure S1b).

To further investigate the effect of combining the inhibitors with radiation compared to either alone, the sphere-forming ability of U87-sph and U251-sph were also tested after treatment. Radiation alone (4 Gy) reduced the number of spheres formed in U87-sph, but not significantly in U251-sph, while the HDACi and MEKi alone at $1 \mu \mathrm{M}$ significantly reduced the number of spheres formed in both (Figure 3a,b and Figure S3). The effect of the inhibitors alone was significantly enhanced in U87-sph when combined with radiation, but not significantly in U251-sph. Additionally, the combination of the HDACi and MEKi (MS-275 and TAK-733 or MS-275 and trametinib) alone at $1 \mu \mathrm{M}$ further significantly reduced the number of spheres formed in both U251-sph and U87-sph compared to the control $(p \leq 0.001$ ) (Figure $3 a, b)$. Upon the addition of 4 Gy radiation, the combined inhibitory effect was more enhanced in U87-sph $(p \leq 0.01)$ compared to U251sph $(p \leq 0.05)$ (Figure 3a,b). Treatment with the standard compound TMZ (at $50 \mu \mathrm{M}$ ) alone or with radiation was more effective in reducing sphere formation in the U87-sph cells compared to the U251-sph cells (Figure 3a,b). Since sphere formation measures the self-renewal of stem-like cells [35-37], these results suggest that the combined treatment of the HDACi and MEKi with radiation could potentially decrease the self-renewal ability of GSLCs.

\subsection{Differential Responses of GSLC Marker Protein Levels to the Combination of HDACi and MEKi with Radiation}

In order to determine whether the combination of the HDAC and MEK inhibitors with radiation was effective against the GSLC marker (Nestin, CD44 and SOX2) protein levels, Western blot quantification was performed $72 \mathrm{~h}$ after the combined treatment.

In U251-sph, radiation alone did not change the protein level of all markers; however, treatment with the HDACi or MEKi alone and with radiation reduced Nestin (Figure 4a). A decrease in SOX2 via treatment with the HDACi MS-275 alone or with radiation was detected, while CD44 was not changed by the compounds alone or with radiation (Figure $4 \mathrm{~b}$ ). Subsequently, the combination of the HDACi and MEKi (MS-275 and TAK-733 or MS275 and trametinib) alone or with radiation significantly eradicated Nestin $(p \leq 0.001)$ and SOX2 $(p \leq 0.05, p \leq 0.001)$ and significantly reduced CD44 $(p \leq 0.05, p \leq 0.01)$ (Figure 4a-d). 
(a)

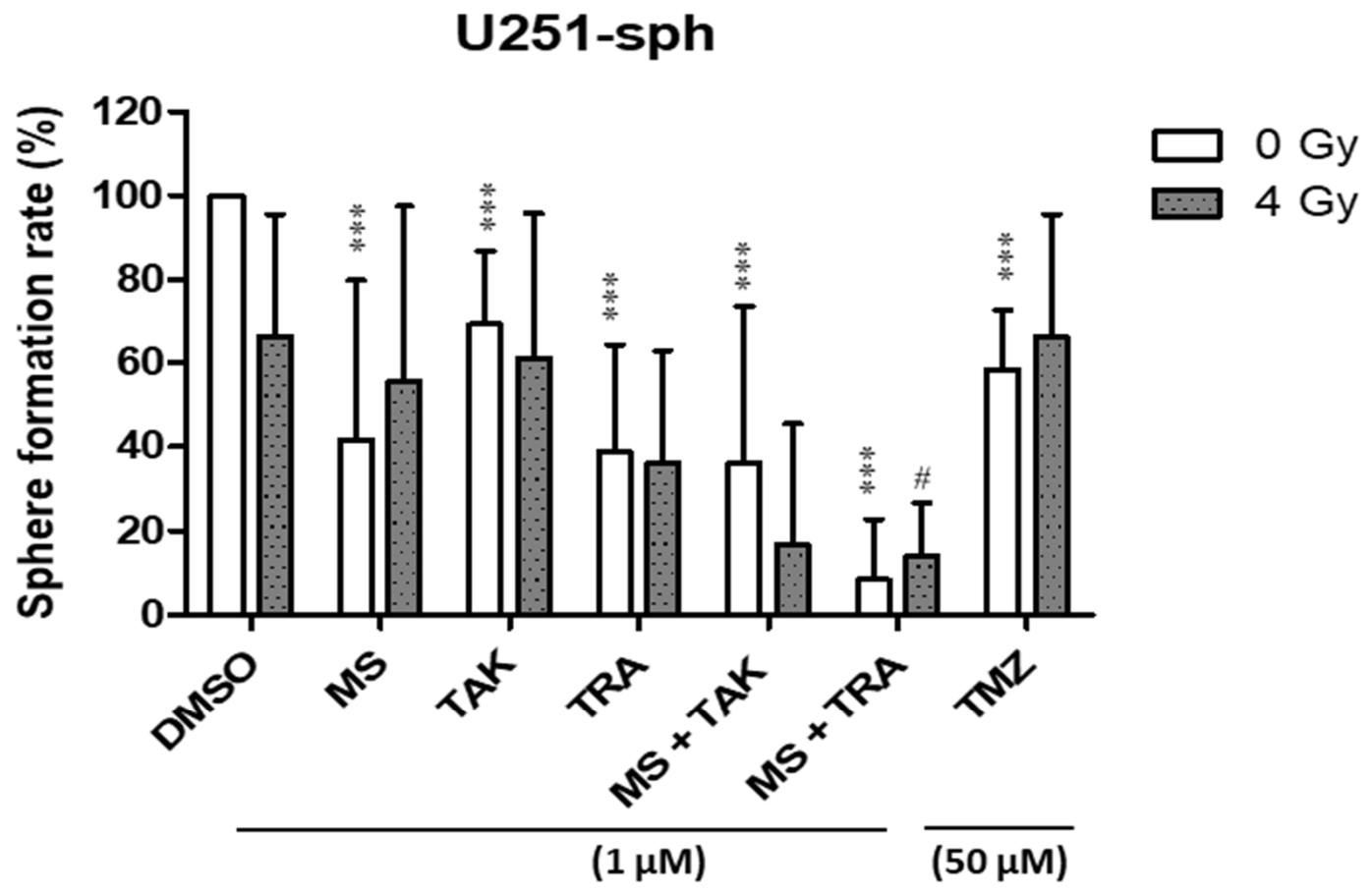

(b)

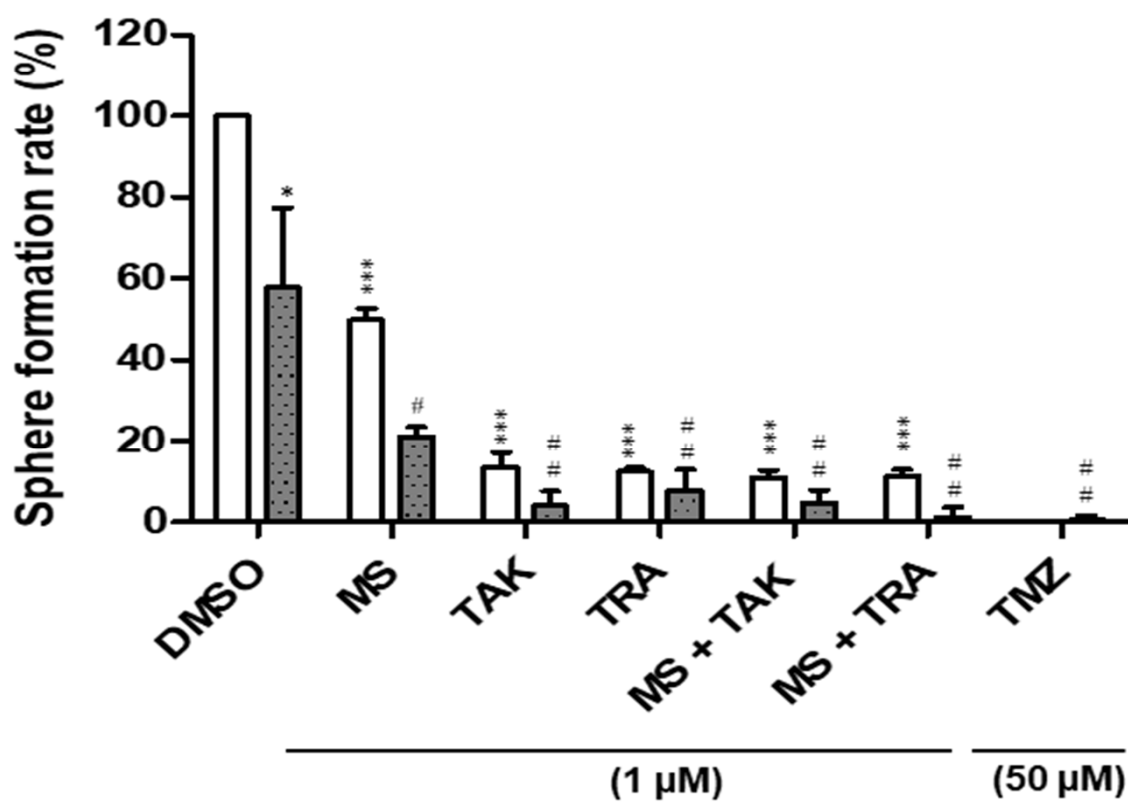

Figure 3. Treatment of HDAC and MEK inhibitors with radiation-inhibited sphere formation of GBderived spheres. (a) Quantification of sphere formation rate (\%) after the treatment of U251-sph and (b) U87-sph with HDCAi (1 $\mu$ M MS-275 (MS)), MEKi (1 $\mu$ M TAK-733 (TAK) or $1 \mu$ M trametinib (TRA)), a combination of both ( $1 \mu \mathrm{M}$ MS-275 $+1 \mu \mathrm{M}$ TAK-733 or $1 \mu \mathrm{M}$ MS-275 $+1 \mu \mathrm{M}$ trametinib) and $50 \mu \mathrm{M}$ TMZ alone or with $4 \mathrm{~Gy}$ radiation. The sphere formation rate was normalized to the DMSO-treated cells as the control, which were set to $100 \%$. Data represent the mean of 3 independent experiments performed in triplicates $(n=3 ; \pm \mathrm{SEM})$. Asterisks indicate significant differences between 0 Gy DMSO and treated samples using Student's $t$-test: ${ }^{*} p \leq 0.05$ and ${ }^{* * *} p \leq 0.001$, while hash symbols indicate significant differences between 4 Gy DMSO and 4 Gy treated samples using Student's $t$-test: \# $p \leq 0.05$ and ${ }^{\# \#} p \leq 0.01$. 
Comparable results were detected in U87-sph, where treatments with either HDACi or MEKi alone and with radiation significantly reduced the protein level of Nestin and SOX2 (Figure 4e,g). Upon combination of both HDACi and MEKi with radiation, the protein level of SOX2 was significantly eradicated $(p \leq 0.01)$, while Nestin remained significantly decreased ( $p \leq 0.001$ ) (Figure $4 \mathrm{e}, \mathrm{g}, \mathrm{h}$ ). Contrary to U251-sph, the CD44 protein level was increased in U87-sph by all the different treatment conditions (Figure 4f). Furthermore, the treatment with the standard compound TMZ alone or with radiation in both cell lines was less effective against the GSLC marker protein levels compared to the combination treatments (Figure $4 \mathrm{a}-\mathrm{h}$ ). Overall, these results suggested that, while combining the HDACi and MEKi with radiation has great potential to reduce the protein levels of GSLC markers, there may be differential effectiveness against CD44 levels.

\subsection{Single and Double Expression of GSLC Markers Reduced by the Combination of HDACi and} MEKi with Radiation

The effects of the combined inhibitor and radiation treatment were subsequently evaluated using flow cytometry analysis of the GSLC markers. Similar to the protein analysis results, only the combination of the HDACi and MEKi (MS-275 and TAK-733 or MS-275 and trametinib) could significantly reduce the Nestin+, CD44+ and SOX2+ populations in U251-sph (Figure 5a,b). However, these decreases in the positive GSLC marker populations were significantly more pronounced when combined with 4 Gy irradiation $(p \leq 0.001)$ (Figure 5a,b and Figure S5). For example, the Nestin+ population in U251-sph decreased from $97 \pm 3 \%$ with 4 Gy irradiation alone to $42 \pm 12 \%$ when treated with MS-275 and TAK-733 alone and a further reduction to $12 \pm 1 \%$ by adding $4 \mathrm{~Gy}$ irradiation to the combination (Figure 5a,b). A similar effect of radiation was also observed with the combination of MS-275 and trametinib in U251-sph (Figure 5a,b).

\section{U251-sph}

(a)

(c)
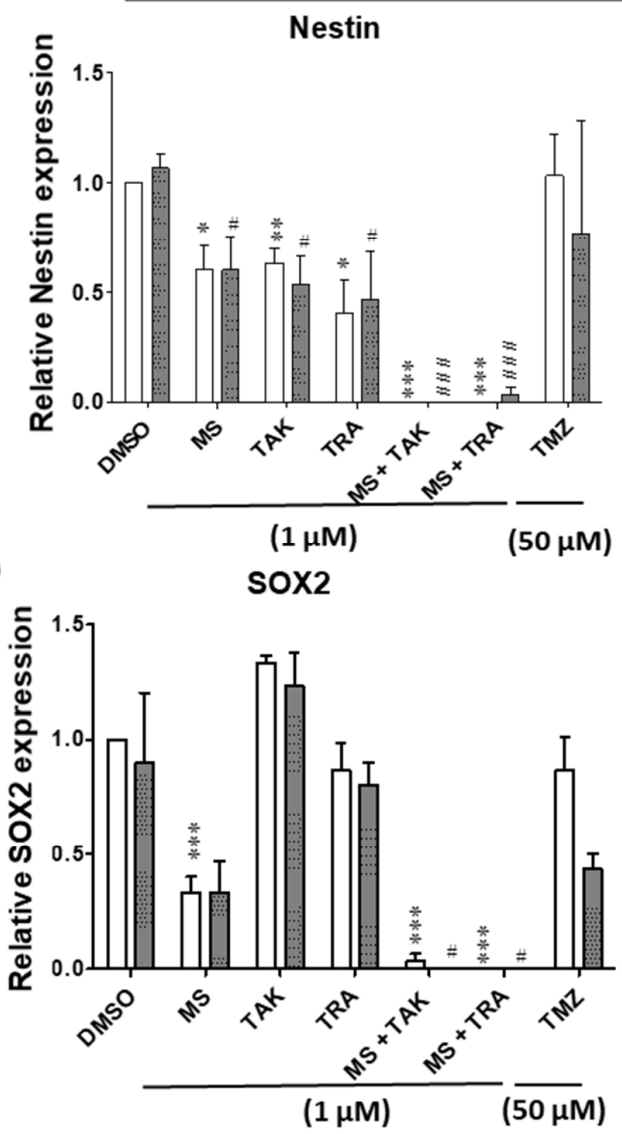

(b)

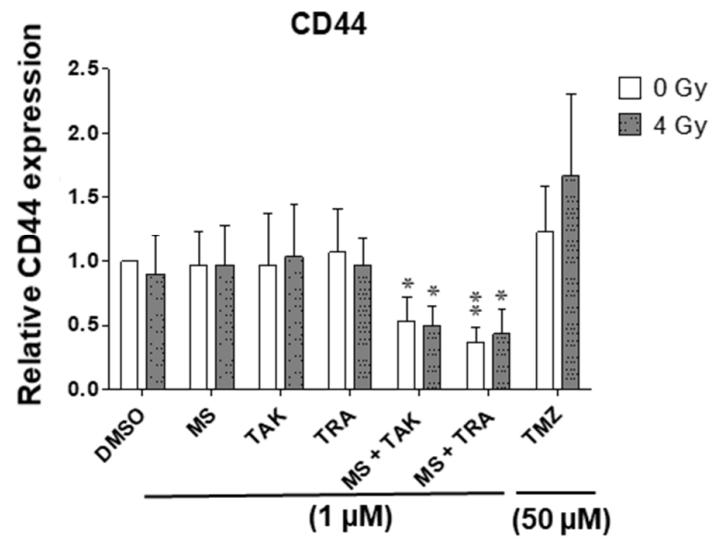

$0 \mathrm{~Gy}$

$4 \mathrm{~Gy}$ (d) DMSO MS TAK TRA MS+TAK MS+TRA TMZ $\begin{array}{lllll}(1 \mu \mathrm{M}) & (1 \mu \mathrm{M}) & (1 \mu \mathrm{M}) & (1 \mu \mathrm{M}) & (1 \mu \mathrm{M})(50 \mu \mathrm{M})\end{array}$

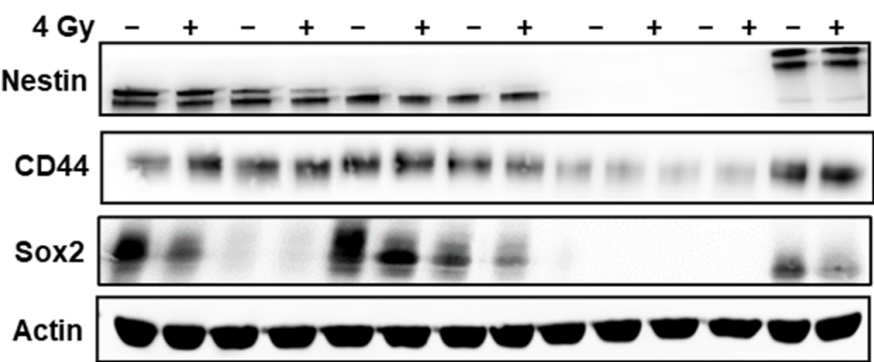

Figure 4. Cont. 
U87-sph

(e)

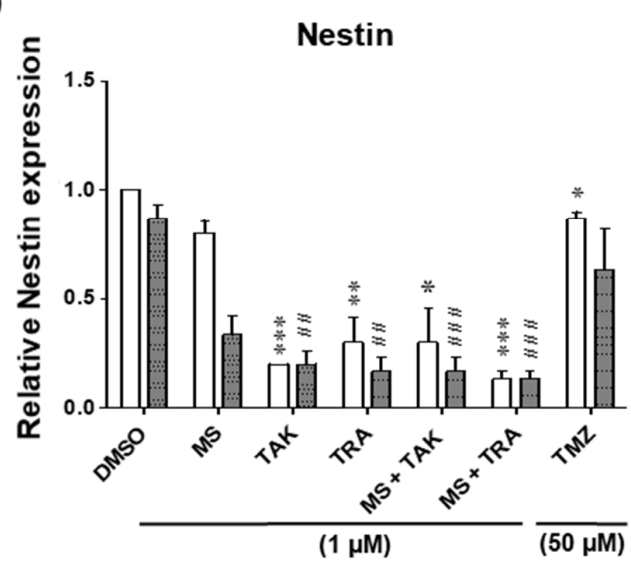

(g)

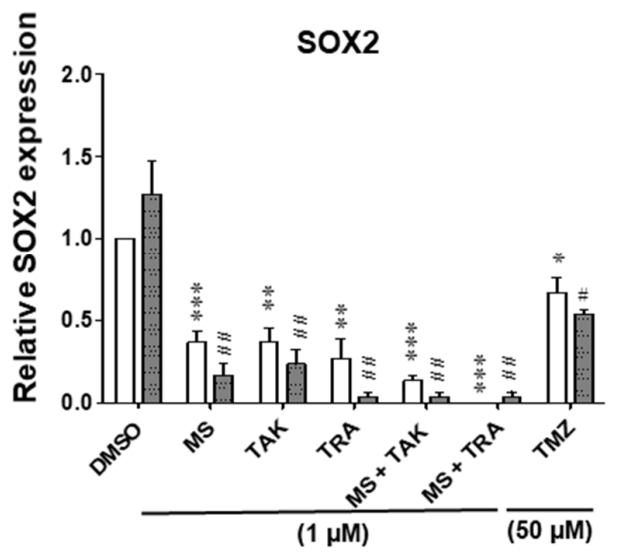

(f)
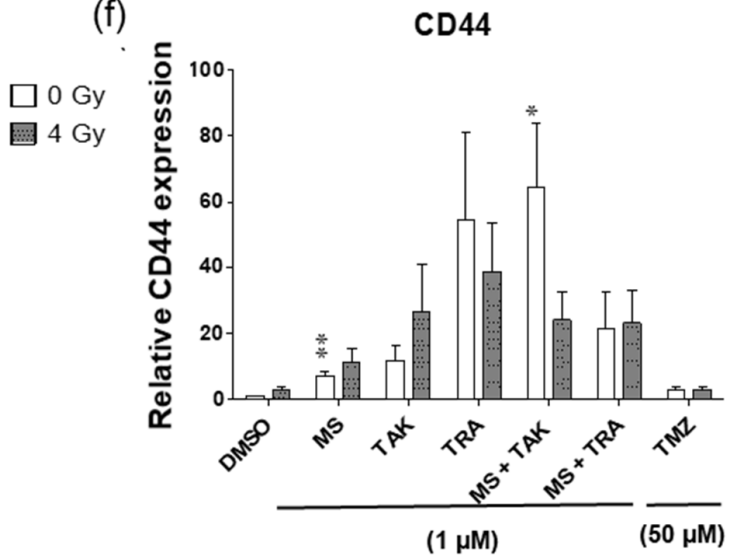

$\square 0 \mathrm{~Gy}$

$4 \mathrm{~Gy}$

(h)

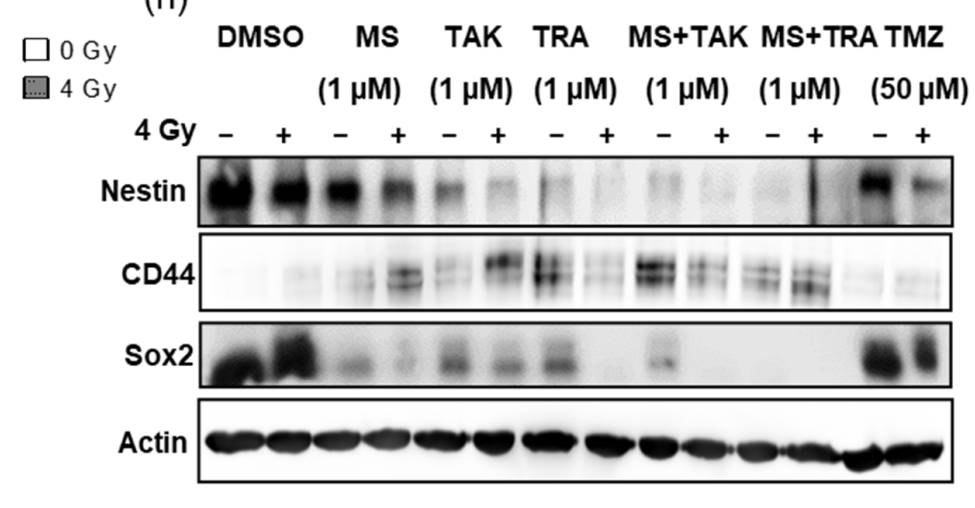

Figure 4. Differential responses of GB-derived spheres to the combination of HDAC and MEK inhibition with radiation. (a) U251-sph protein levels of Nestin, (b) CD44 and (c) SOX2 $72 \mathrm{~h}$ after the spheres were treated with HDCAi $(1 \mu \mathrm{M}$ MS-275), MEKi (1 $\mu$ M TAK-733 or $1 \mu \mathrm{M}$ trametinib), a combination of both ( $1 \mu \mathrm{M}$ MS-275 $+1 \mu \mathrm{M}$ TAK-733 or $1 \mu \mathrm{M}$ MS- $275+1 \mu \mathrm{M}$ trametinib) and $50 \mu \mathrm{M}$ TMZ alone or with 4 Gy radiation. (d) Representative Western blots of GSLC marker protein levels in U251-sph. (e) U87-sph protein levels of Nestin. (f) CD44 and (g) SOX2 after the GSLCs were treated with HDCAi (1 $\mu \mathrm{M}$ MS-275), MEKi (1 $\mu \mathrm{M}$ TAK-733 or $1 \mu \mathrm{M}$ trametinib), a combination of both $(1 \mu \mathrm{M}$ MS-275 $+1 \mu \mathrm{M}$ TAK-733 or $1 \mu \mathrm{M}$ MS-275 $+1 \mu \mathrm{M}$ trametinib) and $50 \mu \mathrm{M}$ TMZ alone or with 4 Gy radiation. (h) Representative Western blots of GSLC marker protein levels in U87-sph. Relative GSLC marker expressions were first normalized to Actin and then to the sham irradiated control cells treated with DMSO. $n=3 ; \pm$ SEM. Asterisks indicate significant differences between 0 Gy DMSO and treated samples using Student's $t$-test: ${ }^{*} p \leq 0.05,{ }^{* *} p \leq 0.01$ and ${ }^{* * *} p \leq 0.001$, while hash symbols indicate significant differences between 4 Gy DMSO and 4 Gy treated samples using Student's $t$-test: ${ }^{\#} p \leq 0.05,{ }^{\# \#} p \leq 0.01$ and ${ }^{\# \# \#} p \leq 0.001$.

In U87-sph, the Nestin+ and SOX2+ populations were significantly decreased by the MEK inhibitors (TAK-733 and trametinib) alone and with 4 Gy irradiation (Figure 5c). Upon the combination of the MEK inhibitors with the HDACi MS-275 and 4 Gy irradiation, a significantly stronger decrease was detected $(p \leq 0.001)$ (Figures $5 \mathrm{c}$ and $\mathrm{S} 7 \mathrm{~b}, \mathrm{c})$. However, CD44 expression was not affected by all treatment conditions in U87-sph (Figures $5 \mathrm{c}$ and $\mathrm{S7a}$ ).

It is now widely accepted that the GSLC population is more accurately identified by the expression of more than one GSLC marker. Since a multicolor approach was used, we further analyzed the co-expression changes of the GSLC markers (CD44+Nestin+, Nestin+SOX2+ and CD44+SOX2+) after the combined treatment with radiation. All three 
double-positive populations of cells were significantly reduced by the MEK inhibitors (TAK-733 and trametinib) alone or in combination with 4 Gy radiation in U87-sph, but not in U251-sph (Figure 6b,c).

(a)

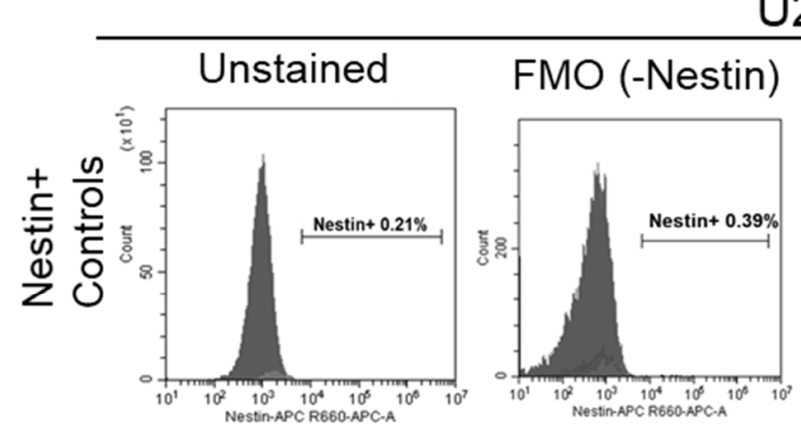

U251-sph
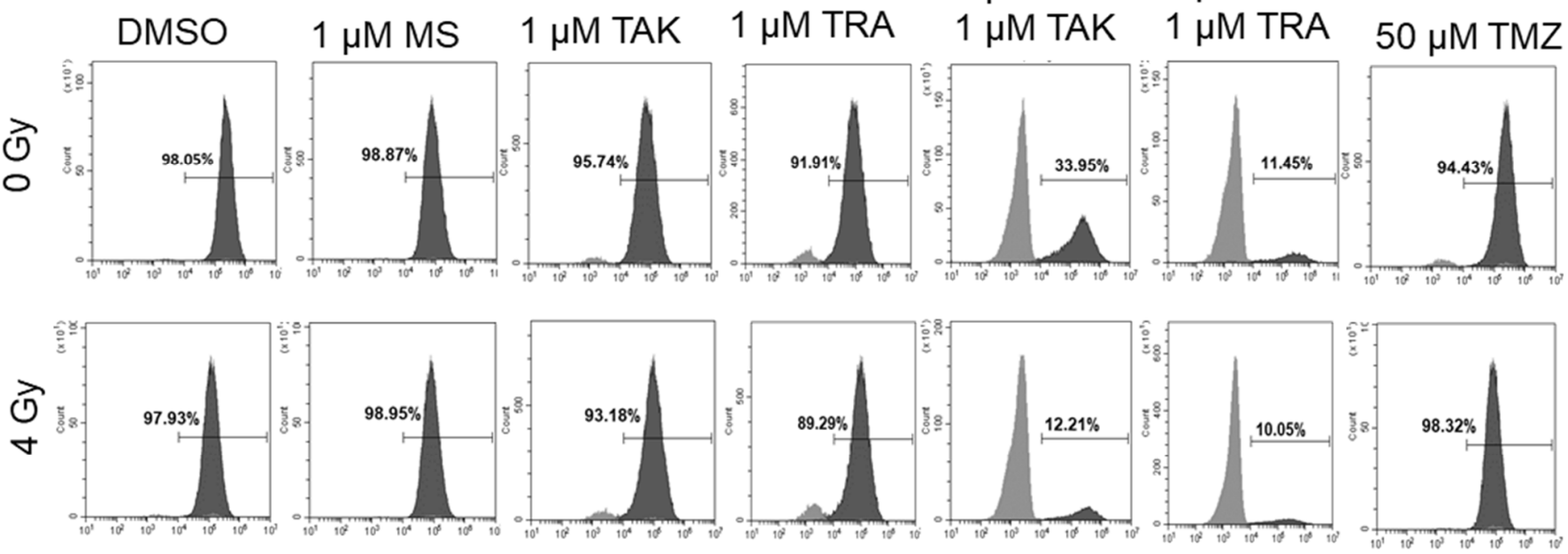

Nestin-APC

(b)

U251-sph

CD44+

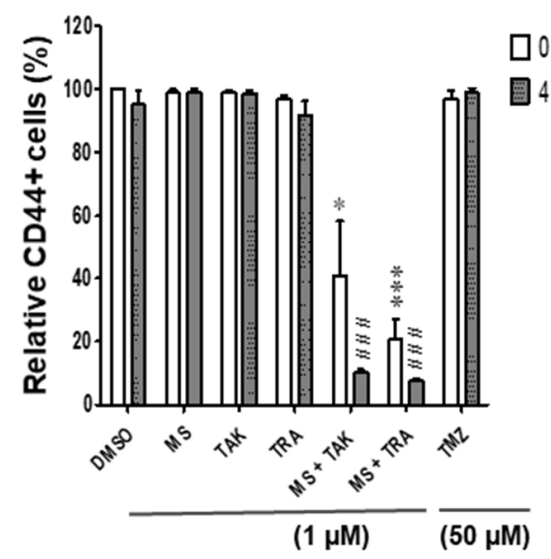

Nestin+

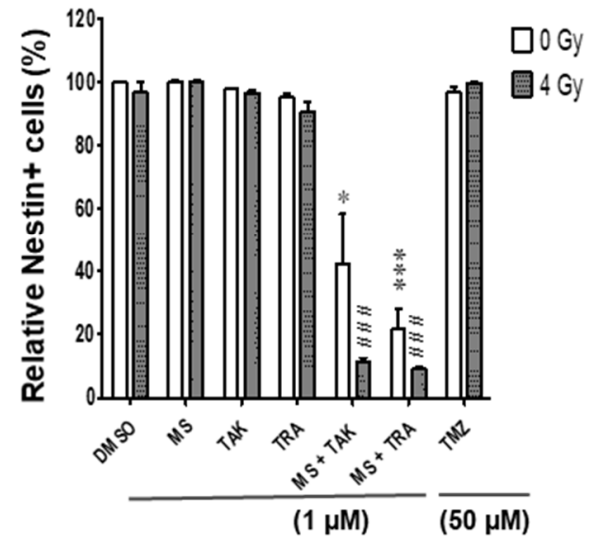

$\operatorname{SOX} 2+$

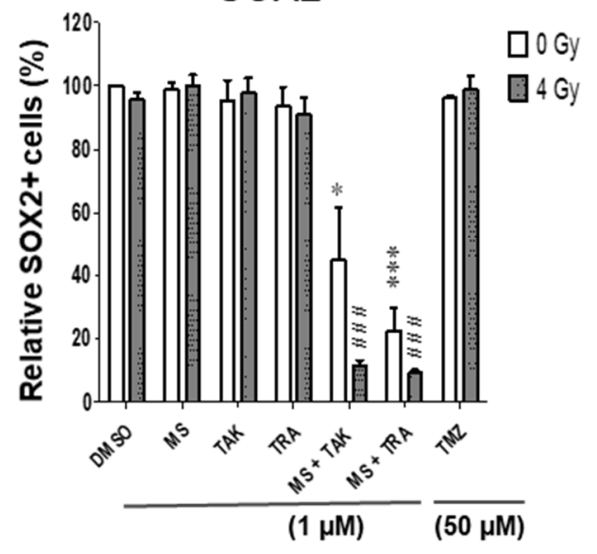

Figure 5. Cont. 
(c) U87-sph

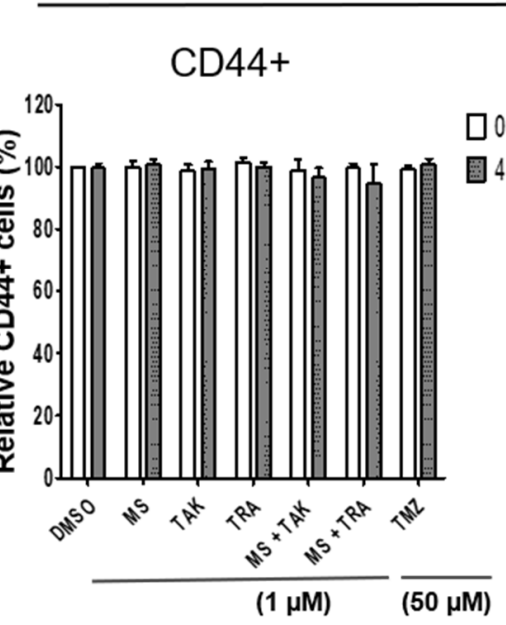

Nestin+

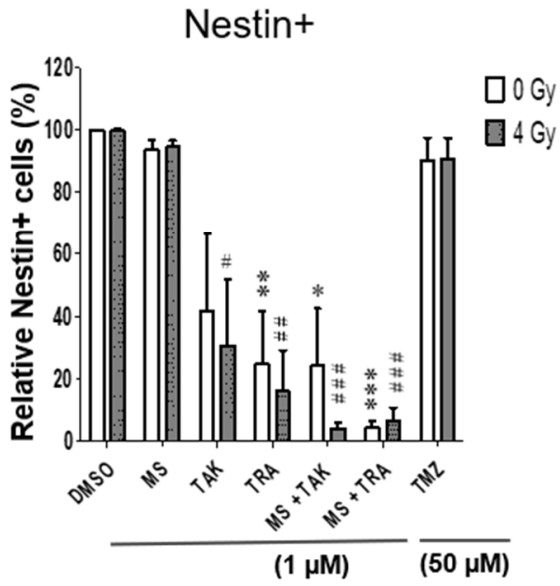

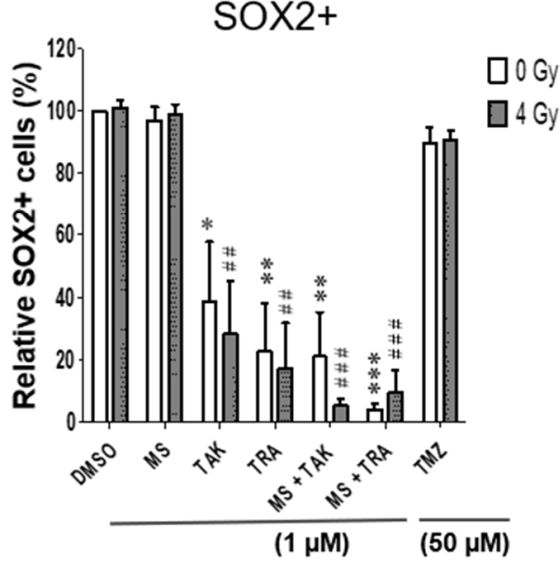

Figure 5. Single expression of GSLC markers reduced by the combination of HDAC and MEK inhibitor with radiation. (a) Representative example of flow cytometric histograms of Nestin in U251-sph $72 \mathrm{~h}$ after the spheres were treated with HDCAi $(1 \mu \mathrm{M}$ MS-275), MEKi $(1 \mu \mathrm{M}$ TAK-733 or $1 \mu \mathrm{M}$ trametinib), a combination of both $(1 \mu \mathrm{M}$ MS-275 $+1 \mu \mathrm{M}$ TAK-733 or $1 \mu \mathrm{M}$ MS-275 $+1 \mu \mathrm{M}$ trametinib) and $50 \mu \mathrm{M} \mathrm{TMZ}$ alone or with $4 \mathrm{~Gy}$ radiation. Upper histograms represent the unstained sample and fluorescence minus one (FMO) control for the gating percentage of Nestin-positive cells (Nestin+). Lower histograms show the percentage of Nestin-positive cells after the indicated treatment conditions and $4 \mathrm{~Gy}$ irradiation. Values inside each histogram represent the percentage of positive single cells from a total of approximately $2 \times 10^{4}$ cells acquired. (b) Quantification of CD44+, Nestin + and SOX2+ relative to sham irradiated control cells (DMSO) set to 100\% in U251-sph and (c) U87-sph. Data represent means $\pm \operatorname{SEM}(n=3)$. Asterisks indicate significant differences between 0 Gy DMSO and treated samples using Student's $t$-test: ${ }^{*} p \leq 0.05,{ }^{* *} p \leq 0.01$ and ${ }^{* * *} p \leq 0.001$, while hash symbols indicate significant differences between $4 \mathrm{~Gy}$ DMSO and $4 \mathrm{~Gy}$ treated samples using Student's $t$-test: ${ }^{\#} p \leq 0.05,{ }^{\#} p \leq 0.01$ and ${ }^{\# \#} p \leq 0.001$.

\section{U251-sph}

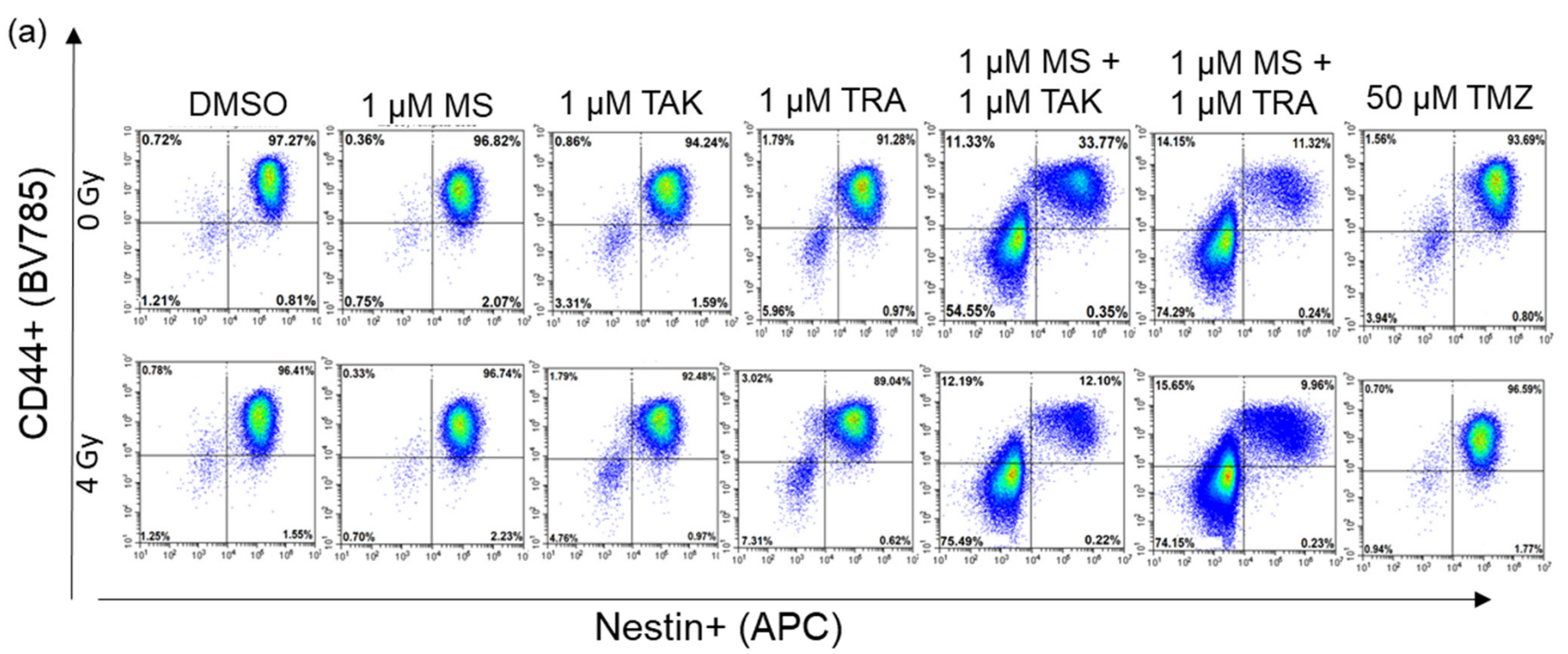

Figure 6. Cont. 
(b)

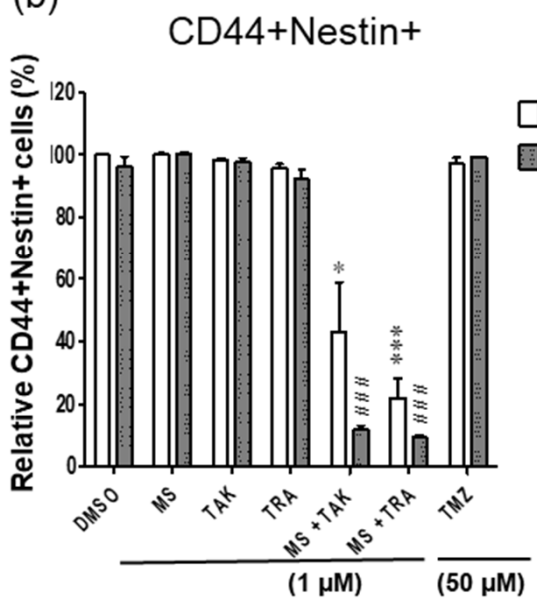

(c)
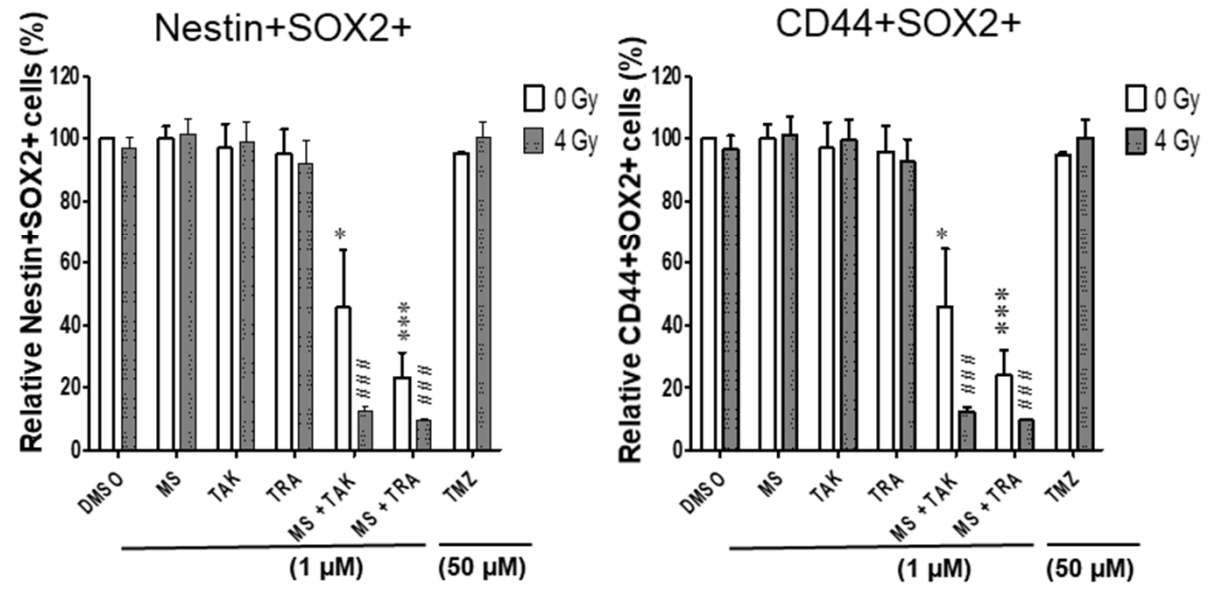

U87-sph
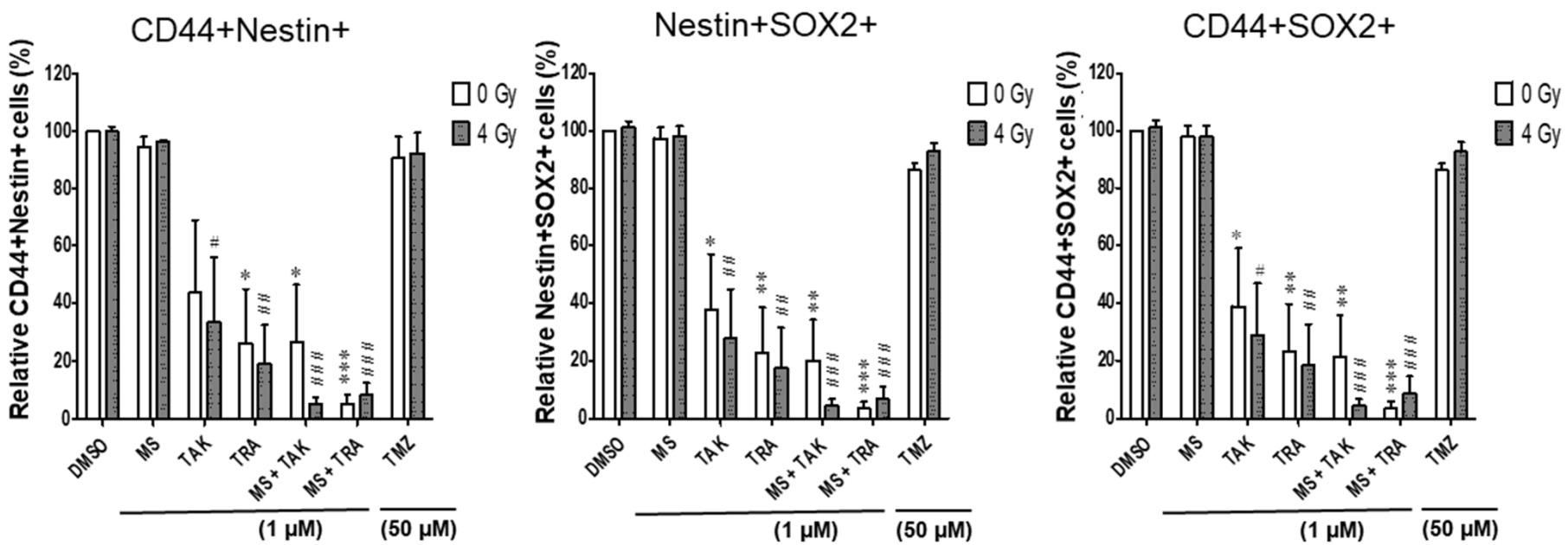

Figure 6. Double expression of GSLC markers reduced by the combination of HDAC and MEK inhibitors with radiation. (a) Representative example of flow cytometric plots in U251-sph $72 \mathrm{~h}$ after the spheres were treated with HDCAi $(1 \mu \mathrm{M}$ MS-275), MEKi $(1 \mu \mathrm{M}$ TAK-733 or $1 \mu \mathrm{M}$ trametinib), a combination of both ( $1 \mu \mathrm{M}$ MS-275 $+1 \mu \mathrm{M}$ TAK-733 or 1 M MS-275 $+1 \mu \mathrm{M}$ trametinib) and $50 \mu \mathrm{M}$ TMZ alone or with 4 Gy radiation. Percentages of double-positive cells for CD44 and Nestin (CD44+Nestin+; upper-right quadrant) after the indicated treatment conditions are shown. Values inside each plot represent the percentage of single cells from a total of approximately $2 \times 10^{4}$ cells acquired. (b) Quantification of CD44+Nestin+, Nestin+SOX2+ and CD44+SOX2+ relative to shamirradiated control cells (DMSO) set to 100\% in U251-sph and (c) U87-sph. Data represent means \pm SEM $(n=3)$. Asterisks indicate significant differences between 0 Gy DMSO and treated samples using Student's $t$-test: ${ }^{*} p \leq 0.05,{ }^{* *} p \leq 0.01$ and ${ }^{* *} p \leq 0.001$, while hash symbols indicate significant differences between 4 Gy DMSO and 4 Gy treated samples using Student's $t$-test: ${ }^{\#} p \leq 0.05,{ }^{\# \#} p \leq 0.01$ and $^{\# \# \#} p \leq 0.001$.

Additionally, combining HDACi and MEKi significantly reduced all double-positive populations in both U251-sph and U87-sph (Figures 6, S6 and S8). However, further exposing the combined inhibitors to 4 Gy radiation significantly enhanced the reduction of all three double-positive populations in both GB-derived spheres $(p \leq 0.001)$ (Figures 6, S6 and S8). This enhanced effect of radiation was more evident in U251-sph than in U87-sph (Figure 6b,c). Again, the standard compound TMZ alone or with 4 Gy radiation was less effective against the single or double expression of the GSLCs markers 
(Figures 5 and 6). Taken together, these results suggested that the combination of HDACi and MEKi with radiation could reduce the GSLC marker expression more efficiently than the standard treatment of TMZ and radiation.

\subsection{Population of Dead Cells Increased by the Combination of HDACi and MEKi with Radiation}

Live-dead staining was performed during flow cytometry antibody staining using a Zombie Aqua dye that is permeant to dead cells due to compromised membranes and non-permeant to live cells. Therefore, it was possible to access the live versus dead status of the cells after the different treatment conditions.

In U251-sph, the dead cell population was not significantly affected by the single treatments of HDACi or MEKi alone and with radiation (Figure 7a). Upon combination, a significant increase in the percentage of dead cells was detected. The dead cells increased from $8 \pm 5 \%$ with only 4 Gy radiation to $58 \pm 16 \%$ with MS-275 and TAK-733 alone and further to $88 \pm 1 \%$ by adding radiation to the combination. Similarly, the percentage of dead cells was $78 \pm 6 \%$ with MS-275 and trametinib alone and further increased to $90 \pm 1 \%$ by including radiation.

Likewise, in U87-sph, the dead cell population was unchanged by single treatments of HDACi and radiation (Figure 7b). Although the MEKi alone and with radiation increased the dead cell population, the highest increase was detected upon the combined treatment of the MEKi and HDACi with radiation.

In contrast, the standard treatment of TMZ and radiation did not significantly affect the dead cell population in both U251-sph and U87-sph.

(a)

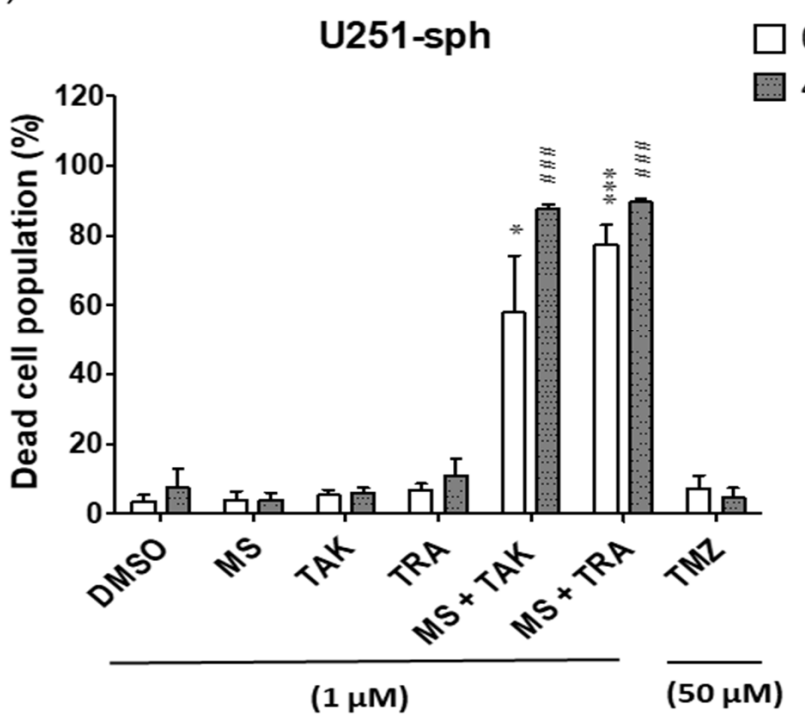

(b)

U87-sph

0 Gy

$4 \mathrm{~Gy}$

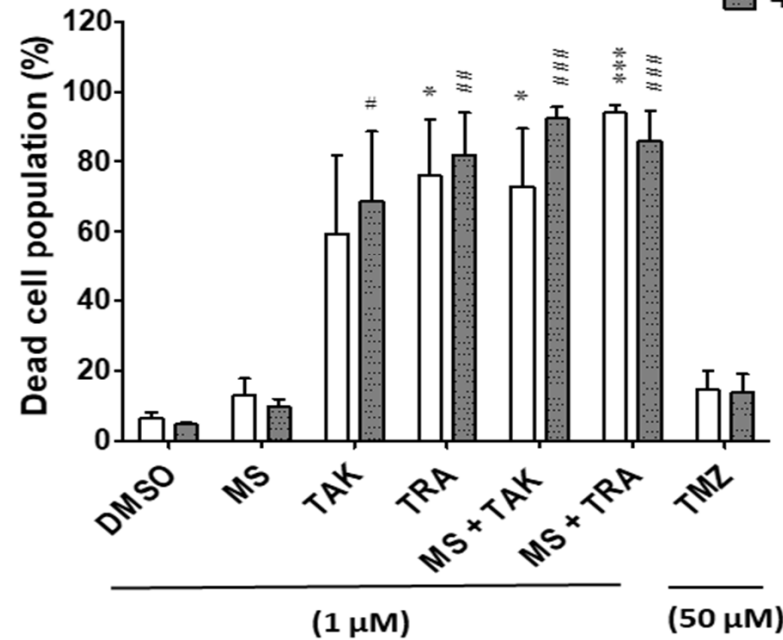

Figure 7. High population of dead cells in GB-derived spheres after treatment with HDACi or MEKi as single or combined compounds with radiation. Quantification data showing the percentage of dead cells in (a) U251-sph and (b) U87-sph after the different treatment conditions. Data represent mean values \pm SEM $(n=3)$. Asterisks indicate significant differences between 0 Gy DMSO and treated samples using Student's $t$-test: ${ }^{*} p \leq 0.05$ and ${ }^{* * *} p \leq 0.001$, while hash symbols indicate significant differences between 4 Gy DMSO and 4 Gy treated samples using Student's $t$-test: ${ }^{\#} p \leq 0.05$, \#\# $p \leq 0.01$ and ${ }^{\# \# \#} p \leq 0.001$.

\section{Discussion}

Radiation plus TMZ remains the most effective non-surgical therapy for GB, although this is mostly palliative due to the radioresistance of GSLCs present within the tumor [2]. Furthermore, GB patients often develop resistance to the DNA-alkylating agent TMZ, along with severe side effects [38]. HDAC and MEK inhibitors have shown promising results as anticancer agents in GB and other tissues $[9,12,39]$. However, they are considered more 
potent when combined with other anticancer agents [40,41]. The combination of HDAC and MEK inhibitors as a chemotherapeutic strategy in tumors was first proposed in a study that showed MEK inhibitors sensitized colon, lung and prostate cancer cells to HDACi-induced cell death [42]. Another study reported that the MEK inhibitors PD184352 or AZD6244 in human colon and lung tumor xenograft models enhanced the efficacy of the HDACi MS-275 and suggested this combination as a promising chemotherapeutic strategy [43]. Subsequently, a Phase 1 study was conducted combining the HDACi MS-275 and the MAPK pathway inhibitor sorafenib to treat patients with solid tumors or acute myeloid leukemia (AML) [44]. This combination increased apoptosis in various cancer cell lines and was well tolerated [44]. As a result, combining HDAC and MEK inhibitors was further investigated in other studies $[16,18,27,28,42,45]$.

MEK inhibition alone in pre-clinical studies of GB displayed antitumor effects, but neither enhanced the efficacy of the standard treatment (radiation or TMZ) nor blocked their effectiveness [46]. HDACis, on the other hand, were proposed as promising anticancer compounds capable of targeting GSLCs in single or combination treatments [47]. The current study, therefore, examined the efficacy of combining an HDACi (MS-275) with MEK inhibitors (TAK-733 or trametinib) via a new approach that includes ionizing radiation as an additional strategy against GB. We employed the spheroid culture method for in vitro enrichment of GSLC markers to induce a stem-like phenotype [48,49] and predict the efficacy of the combined treatment against GSLCs.

The Genomics of Drug Sensitivity in Cancer (GDSC) database revealed that the halfmaximal inhibitory concentration $\left(\mathrm{IC}_{50}\right)$ of the compounds in $\mathrm{GB}$ treatment range from 0.9-51.5 $\mu \mathrm{M}$ for HDACi and 0.1-48.1 $\mu \mathrm{M}$ for MEKi [50]. However, the HDACi and MEKi concentrations were chosen based on the potency of the compounds to reduce the cell viability of the GB-derived spheres at $1 \mu \mathrm{M}$ alone or in combination with $4 \mathrm{~Gy}$ radiation. TMZ, on the other hand, was less effective against the viability of the cells at low concentrations. This was consistent with a study that reported GSLCs to be less sensitive to TMZ treatment via cell viability quantification [51]. Therefore, we decided to use $1 \mu \mathrm{M} \mathrm{HDACi}$ and MEKi, while TMZ was used at a higher concentration of $50 \mu \mathrm{M}$.

The combination of the HDAC and MEK inhibitors (MS-275 and TAK-733 or MS-275 and trametinib), both at a low concentration of $1 \mu \mathrm{M}$, with $4 \mathrm{~Gy}$ radiation decreased the sphere formation rate of the GB-derived spheres. Nevertheless, a higher concentration of TMZ $(50 \mu \mathrm{M})$ with radiation achieved a similar effect. Overall, given that combination therapy is applied to enhance effectiveness, lower doses of the single compounds involved are often desired to lower the risk of drug toxicity to healthy cells [52]. Hence, the efficacy of the HDAC and MEK inhibitors at very low concentrations suggests an improved safety profile and tolerance level for GB therapy as opposed to TMZ [53].

The expressions of GSLC markers CD44, Nestin and SOX2 required for the maintenance of the GSLC population in GB [3] were used to further measure the effect of the combined therapy. All three GSLC markers are reportedly highly expressed in GB compared to normal brain tissues, indicating that they may be responsible for the progression of the tumor and low patient survival rates [54-56]. These GSLC markers were also implicated in GB tumorigenesis and aggressiveness [57]. Therefore, a therapy aimed at downregulating the GSLC markers could be more effective at preventing progression and improving GB treatment [2].

Indeed, we found that combining the HDAC and MEK inhibitors (both at $1 \mu \mathrm{M}$ ) with 4 Gy radiation completely eradicated Nestin and SOX2 protein levels in U251-sph and significantly reduced CD44 protein levels. Similarly, the protein levels of Nestin and SOX2 in U87-sph were also significantly decreased. However, in contrast to U251-sph, the CD44 protein levels were surprisingly upregulated in U87-sph after the combined treatment. This may imply that the sensitivity of CD44 protein levels to the combined HDAC and MEK inhibitor treatment with radiation could be cell line dependent. Similar to our findings, differential responses of protein levels to treatment in the U87-sph and U251-sph were reported [49]. 
Subsequently, the HDAC and MEK inhibitor treatment with radiation strongly reduced the single positive (CD44+, Nestin+ and SOX2+) populations in both cell lines, except for CD44 expression, which remained unchanged in U87-sph. The differential response of CD44 to the combined treatment in both GB-derived spheres could have also been due to the different genetic alterations present in both cell lines.

Since there has been a dispute regarding whether a single GSLC marker expression accurately identifies the GSLC population [57,58], we further evaluated the double expressions of the GSLC markers after the combined treatment. Again, we found that all three double-positive populations (CD44+Nestin+, Nestin+SOX2+ and CD44+SOX2+) were significantly reduced in both GB-derived spheres by the combined HDAC and MEK inhibitor treatment with radiation. Live-dead staining also revealed that the percentage of the dead cell population in both GB-derived spheres was greatly increased upon the combined treatment with radiation. This finding suggested that the mechanism behind the reduced GSLC marker expression after the combined treatment may be due to the killing of the U251-sph and U87-sph cells. While others have shown that the combination of HDACi and MEKi can enhance tumor cell killing compared to either alone [59], our new approach of including radiation shows a further enhancement of this anti-tumor effect in GB. Although more investigations are required, these data suggested that the combined radiochemotherapy may have great potential against the highly resistant GB cells.

Interestingly, the current standard drug TMZ at a higher concentration $(50 \mu \mathrm{M})$, alone or with radiation, either did not have any effect or increased the expression of GSLC markers. In addition, the population of dead cells was not significantly changed compared to the sham irradiated control cells. Some studies have reported TMZ chemoresistance of glioma stem cells and GB [60,61]. Another study reported an increase in the CD44+ population in patient-derived GSLCs in response to TMZ and radiation [51]. An explanation for the chemoresistance to TMZ could be an increased expression of a DNA repair enzyme known as MGMT (O-6-methylguanine-DNA methyltransferase) [62]. MGMT efficiently repairs the DNA damage caused by TMZ, hence only GB cells with an epigenetically silenced expression of the enzyme can benefit from TMZ treatment [63]. Our data has shown that TMZ alone or with radiation was less effective, whereas the combination of the HDAC and MEK inhibitors with radiation efficiently reduced the GSLC markers.

Some reported anti-tumor mechanisms behind the HDAC and MEK inhibitor combination in other cancer studies include enhanced production of ROS (reactive oxygen species) [42], activation of cell-cycle inhibitors [45], increased apoptosis and deregulated survival pathways [59]. In addition to this, we showed that by including the cytotoxic effects of radiation as a novelty, the combined radiochemotherapy treatment could be more effective in the killing of GB tumor cells.

A limitation to our study was that the GB-derived spheres did not fully represent the GSLC population due to the artificial culture conditions, nor did our work establish the combination index of the HDAC and MEK inhibitors. To better elucidate the efficacy of the combined therapy with radiation, further research is necessary using experimental models that involve purely isolated GSLC populations. This can be achieved through fluorescence-activated cell sorting (FACS) to isolate side populations expressing GSLC markers [64] or low-passage patient-derived primary GSLCs [65]. In addition, in vivo validations are required since the in vitro cultures fail to either address the heterogeneity of the original tumor or recapitulate the hierarchy of GSLCs $[3,66]$.

\section{Conclusions}

In conclusion, our findings support the proposition that a combination therapy approach against GB may be an effective way to improve outcomes [40]. The efficacy of this approach was demonstrated by a reduction in the ability of the GB-derived spheres to form spheroids after the combined HDAC and MEK inhibitor (MS-275 and TAK-733 or MS-275 and trametinib) treatment with radiation. The combined treatment with radiation further decreased the expression of the GSLC markers Nestin and SOX2, while the effect on CD44 
was cell line dependent. Moreover, the combined treatment was more efficient compared to the standard treatment of $\mathrm{TMZ}$ and radiation. Although more research is needed to validate the efficacy of this combination strategy, the results suggested that this may be a promising multimodal therapy against the highly resistant GB that can inhibit recurrence and increase the survival of patients.

Supplementary Materials: The following are available online at https:/ /www.mdpi.com/article/10 $.3390 /$ cells11050775/s1. Figure S1: Activities of the HDACi MS-275 (MS) and the MEKi TAK-733 (TAK) or trametinib (TRA) in GSLC lines. (a) Western blots showing the increased acetylation effect of MS-275 on histone H3 in whole-cell lysates from U87-sph and U251-sph cells $(n=2)$. (b) Western blots showing the inhibitory effects of TAK-733 and trametinib on phosphorylated MAPK (pMAPK) in U87-sph and U251-sph cells. Figure S2: Cell viability decreased with increasing concentration of compounds (MS-275, TAK-733, trametinib and TMZ), with and without 4 Gy radiation. (a) Cell viability of U87-sph after the $1 \mu \mathrm{M}$, (b) $10 \mu \mathrm{M}$ and (c) $50 \mu \mathrm{M}$ compound and radiation treatments. (d) Cell viability of U251-sph after the $1 \mu \mathrm{M}$, (e) $10 \mu \mathrm{M}$ and (f) $50 \mu \mathrm{M}$ compound and radiation treatment. Values were normalized to control samples (DMSO) set to 1. Bars not visible represent values less than or equal to 0 . Data represent the mean \pm SEM of three independent experiments performed in quadruplicates $\left(t\right.$-test, $\left.{ }^{* * *} p \geq 0.0001\right)$. Figure S3: Treatment of HDAC and MEK inhibitors with radiation-inhibited sphere formation of GSLC lines. (a) Representative images of spheres formed 14 days after treatment with compounds and radiation in U87-sph cells and (b) U251sph cells (scale bar: $100 \mu \mathrm{m}$ ). Figure S4: Gating strategy and FMO controls for the flow cytometry results shown in Figures 5 and 6. (a) Cells were gated based on size and granularity using side scatter area (SSC-A) vs. forward scatter area (FSC-A) to remove debris and clumped cells. From this cell gate, single cells (singlets) were sub-gated using forward scatter height (FSC-H) vs. FSC-A to remove doublets. From the singlets gates, the control and treated samples were sub-gated to obtain the negative and positive single or double populations. (b) Fluorescence minus one (FMO) controls used to gate the double-positive populations for Figure 6 are shown. The gating region on the FMO controls was set to contain less than $1 \%$ of the cells for double-positive populations. FMO control minus (-) of each indicated antibody is shown. Figure S5: Representative pictures for the single positive populations of (a) CD44+ and (b) SOX2+ in U251-sph for Figure 5b. Unstained, FMO and isotype control (SOX2) for gating positive population are shown. Figure S6: Representative pictures for the double-positive populations of (a) Nestin+SOX2+ and (b) CD44+SOX2+ in U251-sph for Figure 6b. Figure S7: Representative pictures for the single positive populations of (a) CD44+, (b) SOX2+ and (c) Nestin+ in U87-sph for Figure 5c. Unstained, FMO and isotype control (SOX2) for gating positive populations are shown. Values inside each flow cytometric plot represent the percentage of single positive cells from a total of approximately $2 \times 10^{4}$ cells acquired. Figure S8: Representative pictures for the double-positive populations of (a) CD44+Nestin+, (b) Nestin+SOX2+ and (c) CD44+SOX2+ in U87-sph for Figure 6c.

Author Contributions: Conceptualization, M.J.A. and N.A.; methodology, E.I.E., T.P.H., M.J.A. and N.A.; validation, E.I.E., T.P.H., M.J.A. and N.A.; formal analysis, E.I.E., T.P.H., M.J.A. and N.A.; investigation, E.I.E.; resources, E.I.E., T.P.H., M.J.A. and N.A.; data curation, E.I.E. and N.A.; writing-original draft preparation, E.I.E.; writing—review and editing, E.I.E., T.P.H., M.J.A. and N.A.; visualization, E.I.E. and N.A.; supervision, M.J.A. and N.A.; project administration, M.J.A. and N.A.; funding acquisition, M.J.A. and N.A. All authors have read and agreed to the published version of the manuscript.

Funding: This research received no external funding.

Institutional Review Board Statement: Not applicable.

Informed Consent Statement: Not applicable.

Data Availability Statement: Data is contained within the article or supplementary material.

Acknowledgments: The authors thank Kamyar Hadian and Kenji Schorpp for the Operetta Imaging System maintenance and the HMGU Immunoanalytics-Core Facility for support with the flow cytometry experiments. We also thank Michael Rosemann for his advice on the statistical data.

Conflicts of Interest: The authors declare no conflict of interest. 


\section{References}

1. Stupp, R.; Mason, W.P.; van den Bent, M.J.; Weller, M.; Fisher, B.; Taphoorn, M.J.; Belanger, K.; Brandes, A.A.; Marosi, C.; Bogdahn, U.; et al. Radiotherapy plus concomitant and adjuvant temozolomide for glioblastoma. N. Engl. J. Med. 2005, 352, 987-996. [CrossRef] [PubMed]

2. Huang, Z.; Cheng, L.; Guryanova, O.A.; Wu, Q.; Bao, S. Cancer stem cells in glioblastoma-molecular signaling and therapeutic targeting. Protein Cell 2010, 1, 638-655. [CrossRef] [PubMed]

3. Lathia, J.D.; Mack, S.C.; Mulkearns-Hubert, E.E.; Valentim, C.L.L.; Rich, J.N. Cancer stem cells in glioblastoma. Genes Dev. 2015, 29, 1203-1217. [CrossRef] [PubMed]

4. $\quad$ Bao, S.; Wu, Q.; McLendon, R.E.; Hao, Y.; Shi, Q.; Hjelmeland, A.B.; Dewhirst, M.W.; Bigner, D.D.; Rich, J.N. Glioma stem cells promote radioresistance by preferential activation of the DNA damage response. Nature 2006, 444, 756-760. [CrossRef]

5. Yi, Y.; Hsieh, I.-Y.; Huang, X.; Li, J.; Zhao, W. Glioblastoma Stem-Like Cells: Characteristics, Microenvironment, and Therapy. Front. Pharmacol. 2016, 7. [CrossRef]

6. Fremin, C.; Meloche, S. From basic research to clinical development of MEK1/2 inhibitors for cancer therapy. J. Hematol. Oncol. 2010, 3, 8. [CrossRef]

7. Bayin, N.S.; Modrek, A.S.; Placantonakis, D.G. Glioblastoma stem cells: Molecular characteristics and therapeutic implications. World J. Stem Cells 2014, 6, 230-238. [CrossRef]

8. Akinleye, A.; Furqan, M.; Mukhi, N.; Ravella, P.; Liu, D. MEK and the inhibitors: From bench to bedside. J. Hematol. Oncol. 2013, 6, 27. [CrossRef]

9. $\quad$ Perreault, S.; Larouche, V.; Tabori, U.; Hawkin, C.; Lippé, S.; Ellezam, B.; Décarie, J.-C.; Théoret, Y.; Métras, M.-É.; Sultan, S.; et al. A phase 2 study of trametinib for patients with pediatric glioma or plexiform neurofibroma with refractory tumor and activation of the MAPK/ERK pathway: TRAM-01. BMC Cancer 2019, 19, 1250. [CrossRef]

10. de la Puente, P.; Muz, B.; Jin, A.; Azab, F.; Luderer, M.; Salama, N.N.; Azab, A.K. MEK inhibitor, TAK-733 reduces proliferation, affects cell cycle and apoptosis, and synergizes with other targeted therapies in multiple myeloma. Blood Cancer J. 2016, 6, e399. [CrossRef]

11. Cheng, Y.; Tian, H. Current Development Status of MEK Inhibitors. Molecules 2017, 22, 1551. [CrossRef] [PubMed]

12. Anastasov, N.; Hirmer, E.; Klenner, M.; Ott, J.; Falkenberg, N.; Bao, X.; Mutschelknaus, L.; Moertl, S.; Combs, S.; Atkinson, M.J.; et al. MEK1 Inhibitor Combined with Irradiation Reduces Migration of Breast Cancer Cells Including miR-221 and ZEB1 EMT Marker Expression. Cancers 2020, 12, 3760. [CrossRef] [PubMed]

13. Manoharan, N.; Choi, J.; Chordas, C.; Zimmerman, M.A.; Scully, J.; Clymer, J.; Filbin, M.; Ullrich, N.J.; Bandopadhayay, P.; Chi, S.N.; et al. Trametinib for the treatment of recurrent/progressive pediatric low-grade glioma. J. Neuro-Oncol. 2020, 149, 253-262. [CrossRef] [PubMed]

14. Paul, M.R.; Pehlivan, K.C.; Milburn, M.; Yeh-Nayre, L.; Elster, J.; Crawford, J.R. Trametinib-based Treatment of Pediatric CNS Tumors: A Single Institutional Experience. J. Pediatric Hematol. Oncol. 2020, 42, e730-e737. [CrossRef]

15. Adjei, A.A.; LoRusso, P.; Ribas, A.; Sosman, J.A.; Pavlick, A.; Dy, G.K.; Zhou, X.; Gangolli, E.; Kneissl, M.; Faucette, S.; et al. A phase I dose-escalation study of TAK-733, an investigational oral MEK inhibitor, in patients with advanced solid tumors. Invest New Drugs 2017, 35, 47-58. [CrossRef]

16. Carson, R.; Celtikci, B.; Fenning, C.; Javadi, A.; Crawford, N.; Carbonell, L.P.; Lawler, M.; Longley, D.B.; Johnston, P.G.; Van Schaeybroeck, S. HDAC Inhibition Overcomes Acute Resistance to MEK Inhibition in BRAF-Mutant Colorectal Cancer by Downregulation of c-FLIPL. Clin. Cancer Res. Off. J. Am. Assoc. Cancer Res. 2015, 21, 3230-3240. [CrossRef]

17. Thurn, K.T.; Thomas, S.; Moore, A.; Munster, P.N. Rational therapeutic combinations with histone deacetylase inhibitors for the treatment of cancer. Future Oncol 2011, 7, 263-283. [CrossRef]

18. Chao, M.-W.; Chang, L.-H.; Tu, H.-J.; Chang, C.-D.; Lai, M.-J.; Chen, Y.-Y.; Liou, J.-P.; Teng, C.-M.; Pan, S.-L. Combination treatment strategy for pancreatic cancer involving the novel HDAC inhibitor MPT0E028 with a MEK inhibitor beyond K-Ras status. Clin. Epigenetics 2019, 11, 85. [CrossRef]

19. Zhang, G.; Park, M.A.; Mitchell, C.; Hamed, H.; Rahmani, M.; Martin, A.P.; Curiel, D.T.; Yacoub, A.; Graf, M.; Lee, R.; et al. Vorinostat and Sorafenib Synergistically Kill Tumor Cells via FLIP Suppression and CD95 Activation. Clin. Cancer Res. 2008, 14, 5385. [CrossRef]

20. Nagarajan, R.P.; Costello, J.F. Epigenetic mechanisms in glioblastoma multiforme. Semin. Cancer Biol. 2009, 19, 188-197. [CrossRef]

21. Mottamal, M.; Zheng, S.; Huang, T.L.; Wang, G. Histone Deacetylase Inhibitors in Clinical Studies as Templates for New Anticancer Agents. Molecules 2015, 20, 3898-3941. [CrossRef] [PubMed]

22. Sun, P.; Xia, S.; Lal, B.; Eberhart, C.G.; Quinones-Hinojosa, A.; Maciaczyk, J.; Matsui, W.; Dimeco, F.; Piccirillo, S.M.; Vescovi, A.L.; et al. DNER, an epigenetically modulated gene, regulates glioblastoma-derived neurosphere cell differentiation and tumor propagation. Stem Cells 2009, 27, 1473-1486. [CrossRef] [PubMed]

23. Eyupoglu, I.Y.; Hahnen, E.; Trankle, C.; Savaskan, N.E.; Siebzehnrubl, F.A.; Buslei, R.; Lemke, D.; Wick, W.; Fahlbusch, R.; Blumcke, I. Experimental therapy of malignant gliomas using the inhibitor of histone deacetylase MS-275. Mol. Cancer Ther. 2006, 5, 1248-1255. [CrossRef] [PubMed]

24. Chen, R.; Zhang, M.; Zhou, Y.; Guo, W.; Yi, M.; Zhang, Z.; Ding, Y.; Wang, Y. The application of histone deacetylases inhibitors in glioblastoma. J. Exp. Clin. Cancer Res. 2020, 39, 138. [CrossRef] 
25. Bezecny, P. Histone deacetylase inhibitors in glioblastoma: Pre-clinical and clinical experience. Med. Oncol. 2014, $31,985$. [CrossRef]

26. Moertl, S.; Payer, S.; Kell, R.; Winkler, K.; Anastasov, N.; Atkinson, M.J. Comparison of Radiosensitization by HDAC Inhibitors CUDC-101 and SAHA in Pancreatic Cancer Cells. Int. J. Mol. Sci. 2019, 20, 3259. [CrossRef]

27. Nishioka, C.; Ikezoe, T.; Yang, J.; Koeffler, H.P.; Yokoyama, A. Inhibition of MEK/ERK signaling synergistically potentiates histone deacetylase inhibitor-induced growth arrest, apoptosis and acetylation of histone $\mathrm{H} 3$ on p21waf1 promoter in acute myelogenous leukemia cell. Leukemia 2008, 22, 1449-1452. [CrossRef]

28. Torres-Adorno, A.M.; Lee, J.; Kogawa, T.; Ordentlich, P.; Tripathy, D.; Lim, B.; Ueno, N.T. Histone Deacetylase Inhibitor Enhances the Efficacy of MEK Inhibitor through NOXA-Mediated MCL1 Degradation in Triple-Negative and Inflammatory Breast Cancer. Clin. Cancer Res. Off. J. Am. Assoc. Cancer Res. 2017, 23, 4780-4792. [CrossRef]

29. Polivka, J.; Polivka, J.; Holubec, L.; Kubikova, T.; Priban, V.; Hes, O.; Pivovarcikova, K.; Treskova, I. Advances in Experimental Targeted Therapy and Immunotherapy for Patients with Glioblastoma Multiforme. Anticancer Res. 2017, 37, 21-33. [CrossRef]

30. Burster, T.; Traut, R.; Yermekkyzy, Z.; Mayer, K.; Westhoff, M.-A.; Bischof, J.; Knippschild, U. Critical View of Novel Treatment Strategies for Glioblastoma: Failure and Success of Resistance Mechanisms by Glioblastoma Cells. Front. Cell Dev. Biol. 2021, 9. [CrossRef]

31. Tang, Z.; Li, C.; Kang, B.; Gao, G.; Li, C.; Zhang, Z. GEPIA: A web server for cancer and normal gene expression profiling and interactive analyses. Nucleic Acids Res. 2017, 45, W98-W102. [CrossRef]

32. Anastasov, N.; Höfig, I.; Radulović, V.; Ströbel, S.; Salomon, M.; Lichtenberg, J.; Rothenaigner, I.; Hadian, K.; Kelm, J.M.; Thirion, C.; et al. A 3D-microtissue-based phenotypic screening of radiation resistant tumor cells with synchronized chemotherapeutic treatment. BMC Cancer 2015, 15, 466. [CrossRef] [PubMed]

33. Schneider, C.A.; Rasband, W.S.; Eliceiri, K.W. NIH Image to ImageJ: 25 years of image analysis. Nat. Methods 2012, 9, 671-675. [CrossRef]

34. Maecker, H.T.; Trotter, J. Flow cytometry controls, instrument setup, and the determination of positivity. Cytometry. Part A J. Int. Soc. Anal. Cytol. 2006, 69, 1037-1042. [CrossRef] [PubMed]

35. Kanabur, P.; Guo, S.; Simonds, G.R.; Kelly, D.F.; Gourdie, R.G.; Verbridge, S.S.; Sheng, Z. Patient-derived glioblastoma stem cells respond differentially to targeted therapies. Oncotarget 2016, 7, 86406-86419. [CrossRef] [PubMed]

36. Yuan, X.; Curtin, J.; Xiong, Y.; Liu, G.; Waschsmann-Hogiu, S.; Farkas, D.L.; Black, K.L.; Yu, J.S. Isolation of cancer stem cells from adult glioblastoma multiforme. Oncogene 2004, 23, 9392-9400. [CrossRef]

37. Fukaya, R.; Ohta, S.; Yamaguchi, M.; Fujii, H.; Kawakami, Y.; Kawase, T.; Toda, M. Isolation of cancer stem-like cells from a side population of a human glioblastoma cell line, SK-MG-1. Cancer Lett. 2010, 291, 150-157. [CrossRef]

38. Trinh, V.A.; Patel, S.P.; Hwu, W.-J. The safety of temozolomide in the treatment of malignancies. Expert Opin. Drug Saf. 2009, 8 , 493-499. [CrossRef]

39. Camphausen, K.; Scott, T.; Sproull, M.; Tofilon, P.J. Enhancement of Xenograft Tumor Radiosensitivity by the Histone Deacetylase Inhibitor MS-275 and Correlation with Histone Hyperacetylation. Clin. Cancer Res. 2004, 10, 6066. [CrossRef]

40. Ghosh, D.; Nandi, S.; Bhattacharjee, S. Combination therapy to checkmate Glioblastoma: Clinical challenges and advances. Clin Transl. Med. 2018, 7, 33. [CrossRef]

41. Suraweera, A.; O'Byrne, K.J.; Richard, D.J. Combination Therapy with Histone Deacetylase Inhibitors (HDACi) for the Treatment of Cancer: Achieving the Full Therapeutic Potential of HDACi. Front. Oncol. 2018, 8, 92. [CrossRef] [PubMed]

42. Ozaki, K.; Minoda, A.; Kishikawa, F.; Kohno, M. Blockade of the ERK pathway markedly sensitizes tumor cells to HDAC inhibitor-induced cell death. Biochem. Biophys. Res. Commun. 2006, 339, 1171-1177. [CrossRef] [PubMed]

43. Sakamoto, T.; Ozaki, K.-i.; Fujio, K.; Kajikawa, S.-h.; Uesato, S.-i.; Watanabe, K.; Tanimura, S.; Koji, T.; Kohno, M. Blockade of the ERK pathway enhances the therapeutic efficacy of the histone deacetylase inhibitor MS-275 in human tumor xenograft models. Biochem. Biophys. Res. Commun. 2013, 433, 456-462. [CrossRef] [PubMed]

44. Ngamphaiboon, N.; Dy, G.; Ma, W.W.; Zhao, Y.; Reungwetwattana, T.; DePaolo, D.; Ding, Y.; Brady, W.; Fetterly, G.; Adjei, A. A phase I study of the histone deacetylase (HDAC) inhibitor entinostat, in combination with sorafenib in patients with advanced solid tumors. Investig. New Drugs 2014, 33, 225-232. [CrossRef] [PubMed]

45. Yamada, T.; Amann, J.M.; Tanimoto, A.; Taniguchi, H.; Shukuya, T.; Timmers, C.; Yano, S.; Shilo, K.; Carbone, D.P. Histone Deacetylase Inhibition Enhances the Antitumor Activity of a MEK Inhibitor in Lung Cancer Cells Harboring RAS Mutations. Mol. Cancer Ther. 2018, 17, 17-25. [CrossRef]

46. Selvasaravanan, K.D.; Wiederspohn, N.; Hadzalic, A.; Strobel, H.; Payer, C.; Schuster, A.; Karpel-Massler, G.; Siegelin, M.D.; Halatsch, M.-E.; Debatin, K.-M.; et al. The limitations of targeting MEK signalling in Glioblastoma therapy. Sci. Rep. 2020, 10, 7401. [CrossRef]

47. Reddy, R.G.; Bhat, U.A.; Chakravarty, S.; Kumar, A. Advances in histone deacetylase inhibitors in targeting glioblastoma stem cells. Cancer Chemother. Pharmacol. 2020, 86, 165-179. [CrossRef]

48. Zhang, S.; Xie, R.; Wan, F.; Ye, F.; Guo, D.; Lei, T. Identification of U251 glioma stem cells and their heterogeneous stem-like phenotypes. Oncol. Lett. 2013, 6, 1649-1655. [CrossRef]

49. Liu, J.; Liu, Y.; Xie, T.; Luo, L.; Xu, C.; Gao, Q.; Shen, L.; Wan, F.; Lei, T.; Ye, F. Radiation-induced G2/M arrest rarely occurred in glioblastoma stem-like cells. Int. J. Radiat. Biol. 2018, 94, 394-402. [CrossRef] 
50. Yang, W.; Soares, J.; Greninger, P.; Edelman, E.J.; Lightfoot, H.; Forbes, S.; Bindal, N.; Beare, D.; Smith, J.A.; Thompson, I.R.; et al. Genomics of Drug Sensitivity in Cancer (GDSC): A resource for therapeutic biomarker discovery in cancer cells. Nucleic Acids Res. 2012, 41, D955-D961. [CrossRef]

51. Brown, D.V.; Filiz, G.; Daniel, P.M.; Hollande, F.; Dworkin, S.; Amiridis, S.; Kountouri, N.; Ng, W.; Morokoff, A.P.; Mantamadiotis, T. Expression of CD133 and CD44 in glioblastoma stem cells correlates with cell proliferation, phenotype stability and intra-tumor heterogeneity. PLoS ONE 2017, 12, e0172791. [CrossRef] [PubMed]

52. Bayat Mokhtari, R.; Homayouni, T.S.; Baluch, N.; Morgatskaya, E.; Kumar, S.; Das, B.; Yeger, H. Combination therapy in combating cancer. Oncotarget 2017, 8, 38022-38043. [CrossRef]

53. Chamberlain, M.C. Temozolomide: Therapeutic limitations in the treatment of adult high-grade gliomas. Expert Rev. Neurother. 2010, 10, 1537-1544. [CrossRef] [PubMed]

54. Kaaijk, P.; Troost, D.; Morsink, F.; Keehnen, R.M.; Leenstra, S.; Bosch, D.A.; Pals, S.T. Expression of CD44 splice variants in human primary brain tumors. J. Neuro-Oncol. 1995, 26, 185-190. [CrossRef]

55. Schmitz, M.; Temme, A.; Senner, V.; Ebner, R.; Schwind, S.; Stevanovic, S.; Wehner, R.; Schackert, G.; Schackert, H.K.; Fussel, M.; et al. Identification of SOX2 as a novel glioma-associated antigen and potential target for T cell-based immunotherapy. Br. J. Cancer 2007, 96, 1293-1301. [CrossRef]

56. Zhang, M.; Song, T.; Yang, L.; Chen, R.; Wu, L.; Yang, Z.; Fang, J. Nestin and CD133: Valuable stem cell-specific markers for determining clinical outcome of glioma patients. J. Exp. Clin. Cancer Res. 2008, 27, 85. [CrossRef] [PubMed]

57. Bradshaw, A.; Wickremsekera, A.; Tan, S.T.; Peng, L.; Davis, P.F.; Itinteang, T. Cancer Stem Cell Hierarchy in Glioblastoma Multiforme. Front. Surg. 2016, 3, 21. [CrossRef]

58. Lee, G.; Auffinger, B.; Guo, D.; Hasan, T.; Deheeger, M.; Tobias, A.L.; Kim, J.Y.; Atashi, F.; Zhang, L.; Lesniak, M.S.; et al Dedifferentiation of Glioma Cells to Glioma Stem-like Cells By Therapeutic Stress-induced HIF Signaling in the Recurrent GBM Model. Mol. Cancer Ther. 2016, 15, 3064. [CrossRef] [PubMed]

59. Corno, C.; Arrighetti, N.; Ciusani, E.; Corna, E.; Carenini, N.; Zaffaroni, N.; Gatti, L.; Perego, P. Synergistic Interaction of Histone Deacetylase 6- and MEK-Inhibitors in Castration-Resistant Prostate Cancer Cells. Front. Cell Dev. Biol. 2020, 8. [CrossRef]

60. Yamada, R.; Nakano, I. Glioma stem cells: Their role in chemoresistance. World Neurosurg. 2012, 77, 237-240. [CrossRef]

61. Hombach-Klonisch, S.; Mehrpour, M.; Shojaei, S.; Harlos, C.; Pitz, M.; Hamai, A.; Siemianowicz, K.; Likus, W.; Wiechec, E.; Toyota, B.D.; et al. Glioblastoma and chemoresistance to alkylating agents: Involvement of apoptosis, autophagy, and unfolded protein response. Pharmacol. Ther. 2018, 184, 13-41. [CrossRef]

62. Liu, G.; Yuan, X.; Zeng, Z.; Tunici, P.; Ng, H.; Abdulkadir, I.R.; Lu, L.; Irvin, D.; Black, K.L.; Yu, J.S. Analysis of gene expression and chemoresistance of CD133+ cancer stem cells in glioblastoma. Mol. Cancer 2006, 5, 67. [CrossRef] [PubMed]

63. Wick, W.; Weller, M.; van den Bent, M.; Sanson, M.; Weiler, M.; von Deimling, A.; Plass, C.; Hegi, M.; Platten, M.; Reifenberger, G. MGMT testing - the challenges for biomarker-based glioma treatment. Nat. Rev. Neurol. 2014, 10, 372-385. [CrossRef] [PubMed]

64. Inocencio, J.; Frenster, J.D.; Placantonakis, D.G. Isolation of Glioblastoma Stem Cells with Flow Cytometry. In Glioblastoma: Methods and Protocols; Placantonakis, D.G., Ed.; Springer: New York, NY, USA, 2018; pp. 71-79.

65. Dundar, T.T.; Hatiboglu, M.A.; Ergul, Z.; Seyithanoglu, M.H.; Sozen, E.; Tuzgen, S.; Kaynar, M.Y.; Karaoz, E. Glioblastoma Stem Cells and Comparison of Isolation Methods. J. Clin. Med. Res. 2019, 11, 415-421. [CrossRef] [PubMed]

66. Pastrana, E.; Silva-Vargas, V.; Doetsch, F. Eyes wide open: A critical review of sphere-formation as an assay for stem cells. Cell Stem Cell 2011, 8, 486-498. [CrossRef] 